\title{
Spectrum Resource Allocation Based on Cooperative NOMA With Index Modulation
}

\author{
Xuan Chen, Miaowen Wen, Senior Member, IEEE, Tianqi Mao, and Shuping Dang, Member, IEEE
}

\begin{abstract}
In this paper, two novel spectrum resource allocation schemes, based on orthogonal frequency division multiplexing with index modulation (OFDM-IM) and dual-mode OFDMIM, are proposed for a three-node cooperative non-orthogonal multiple access (C-NOMA) system. In the first scheme, we allocate IM bits to serve the cell-edge user, and save the transmit power to assist the delivery of constellation symbols. Alternatively, constrained by the spectral efficiency (SE), the rest information bits of the cell-edge user can be carried by the conventional signal constellation. In the second scheme, to further eliminate the interference between users and boost the $\mathrm{SE}$, different modulation modes (Mode I and Mode II) are employed to distinguish the subcarriers of the cell-center and cell-edge users, and to encode the incoming bit stream from these users, respectively. Furthermore, we consider two different detectors for the cell-edge user, i.e., the optimal/suboptimal maximumlikelihood detectors. Asymptotically tight bounds on the bit error rate of the above-mentioned users are derived in closed-form. Finally, simulation results verify the theoretical analysis and show that the proposed schemes have the potential to outperform $\mathrm{C}$ NOMA.
\end{abstract}

Index Terms-Bit error rate, orthogonal frequency division multiplexing, index modulation, dual-mode, non-orthogonal multiple access, cooperative relaying.

\section{INTRODUCTION}

$\mathrm{W}$ ITH the growth of wireless data traffic arising from the access of a large number of devices and various applications [1], [2], the future wireless networks (5G and beyond) are expected to not only support highly reliable transmission but also ensure the characteristics of high data rate and low collision [3]. In order to meet these demands, a number of multiple access techniques have been carried

Manuscript received December 1, 2019; revised March 23, 2020; accepted April 17, 2020. Date of publication XXXX XX, 2020; date of current version XXXX XX, 2020. This work was supported in part by the Natural Science Foundation of Guangdong Province under Grant 2018B030306005, in part by the Pearl River Nova Program of Guangzhou under Grant 201806010171, in part by the National Natural Science Foundation of China under Grant 61871190 , and in part by the open research fund of the National Mobile Communications Research Laboratory, Southeast University under Grant 2020D03. (Corresponding author: Miaowen wen.)

Xuan Chen is with the School of Electronic and Information Engineering, South China University of Technology, Guangzhou, China (email: eechenxuan@mail.scut.edu.cn)

Miaowen Wen is with the School of Electronic and Information Engineering, South China University of Technology, Guangzhou 510640, China, and also with the National Mobile Communications Research Laboratory, Southeast University, Nanjing 210096, China (e-mail: eemwwen@scut.edu.cn).

Tianqi Mao is with the Beijing National Research Center for Information Science and Technology, Department of Electronic Engineering, Tsinghua University, Beijing 100084, China (email: maotq18@mails.tsinghua.edu.cn).

Shuping Dang is with Computer, Electrical and Mathematical Sciences, and Engineering Division, King Abdullah University of Science and Technology, Thuwal 23955-6900, Saudi Arabia (e-mail: shuping.dang@kaust.edu.sa). out, among which non-orthogonal multiple access (NOMA) is widely discussed and investigated. Compared with conventional orthogonal multiple access (OMA), NOMA can achieve lower latency, a higher system throughput, and a greater degree of fairness [4], [5].

The rationale behind NOMA is to serve multiple users in the same frequency band and time slot with different power levels. This approach inherently requires the NOMA system to perform the superposition coding at the transmitting side and the successive interference cancellation (SIC) at the receiving side, so as to successfully decode the message from different users. As a result, the cell-center user is always required to recover the messages intended for other cell-edge users during the SIC phase. Based on this feature, the cooperative NOMA (C-NOMA) is proposed for fully exploiting prior information known by the cell-center user and further boosting the system performance [6]. Specifically, the cell-center user can act as a decode-and-forward relay to retransmit the information of the cell-edge user, thereby enabling the cell-edge user to obtain a cooperative diversity gain and improve transmission reliability. On the other side, C-NOMA has greatly alleviated the defect that due to a long transmission distance, the celledge users always suffer from poor communication services. Following the paradigm of C-NOMA, Liu et al. [7] applied simultaneous wireless information and power transfer (SWIPT) to C-NOMA, thus alleviating practical constraints on energy consumption. In [8]-[10], the capacity, the receiver design, and the error performance of a three-node C-NOMA system consisting of one base station (BS) and two users (the cellcenter and cell-edge users) have been investigated. Furthermore, the authors of [11] and [12] analyzed the performance of C-NOMA over the Rician and Nakagami- $m$ fading channels, respectively. However, it cannot be ignored that C-NOMA is always susceptible to the inter-user interference (IUI) arising from the multiplexing in the power domain. Further, the high detection complexity caused by SIC also imposes challenges on practical implementations of C-NOMA.

In order to effectively resolve these issues, the spatial modulation (SM) was introduced to a three node C-NOMA network in [13] and [14], thus forming the SM-aided C-NOMA. Specifically, in SM-aided C-NOMA, the information of two users can be independently carried by different informationbearing units such that the complex SIC procedure and IUI can be avoided from the space domain. SM is a typical index modulation (IM) technique, whose core idea is to employ indices of transmit antennas to convey the extra information on the basis of conventional multiple-input multiple-output framework [15]-[17]. There are, however, still some scenarios 
where SM is not convenient or appropriate due to the limitation in physical size, such as sensor nodes, handled terminals, etc. As we well know, the frequency band can be divided into different sub-channels without occupying the actual space. Therefore, it maybe feasible to overcome the major weakness of C-NOMA from the frequency perspective. Among the IM family, orthogonal frequency division multiplexing with index modulation (OFDM-IM) is another classical representative besides SM, which is generated by applying the concept of IM into the OFDM framework [18], [19]. In particular, a fraction of subcarriers in OFDM-IM are activated to carry $M$ ary modulated symbols, whist the others are unutilized. Then, indices of activated subcarriers are capable of conveying extra information bits (a.k.a. IM bits). Due to the existence of silent subcarriers, some transmit power can be saved to assist in conveying $M$-ary symbols. Moreover, the transmission of IM bits not only consumes no additional power or bandwidth, but also has a highly stronger protection mechanism than that of conventional modulation bits [20]. However, constrained by the idle subcarriers, the main bottleneck of OFDM-IM is the limited SE. Consequently, dual-mode OFDM-IM (DMOFDM-IM), as an extension of OFDM-IM, was proposed to further boost the SE, in which all OFDM subcarriers are activated for data transmission with two distinguishable constellation alphabets $M_{A}$ and $M_{B}$, and additional IM bits can be conveyed implicitly by indices of subcarriers modulated by $M_{A}$ [21], [22].

Recently, the application of IM techniques-based on OFDM to the conventional NOMA has been researched in [23] and [24]. In [23], the NOMA based on the OFDM-IM technique was proposed in order to reduce the impact of the collision on ultra-reliable and low-latency communications. The authors of [24] combined NOMA with OFDM-IM to superimpose the index of subcarrier activation pattern of multiple users in the power domain. However, similar to conventional NOMA systems, [23] and [24] cannot alleviate the major defects of NOMA, i.e., the complex SIC and IUI; on the other hand, the prior information decoded by the cell-center user cannot be fully exploited. Therefore, how to effectively avoid the impact of the IUI and the imperfect SIC, while fully exploring the prior information obtained by the cell-center user, is still an open topic, when the BS employs IM techniques-based on OFDM for information transmission.

Against the background, in this paper, we propose two novel three-node C-NOMA networks with OFDM-IM and DM-OFDM-IM, termed as generalized CIM-NOMA (GCIMNOMA) and CDM-NOMA. The considered networks consist of one BS and two users, i.e., a cell-center user and a cell-edge user. The main contributions of this paper are summarized as follows:

1) A novel GCIM-NOMA scheme is proposed. In this scheme, we first allocate IM bits to serve the cell-edge user so as to ensure an adequate communication quality. On the other side, the interference among users caused by the power allocation mechanism can be reduced. Meanwhile, the transmit power saved by deactivating some subcarriers can effectively assist the transmission of constellation symbols. In the cooperative phase, the
TABLE I

SUMMARY OF ACRONYMS

\begin{tabular}{|c|c|}
\hline Acronym & Vocabulary \\
\hline AWGN & Additive white Gaussian noise \\
\hline BS & Base station \\
\hline BER & Bit error rate \\
\hline BEP & Bit error probability \\
\hline bpcu & bits per channel use \\
\hline $\mathrm{CP}$ & Cyclic prefix \\
\hline CR & Cognitive radio \\
\hline C-NOMA & Cooperative NOMA \\
\hline DM & Dual-mode \\
\hline $\mathrm{EE}$ & Energy efficiency \\
\hline GD & Greedy-detector \\
\hline IM & Index modulation \\
\hline IEP & Index error probability \\
\hline IUI & Inter-user interference \\
\hline ML & Maximum-likelihood \\
\hline MRC & Maximal ratio combining \\
\hline NOMA & Non-orthogonal multiple access \\
\hline OMA & Orthogonal multiple access \\
\hline OFDM & Orthogonal frequency division multiplexing \\
\hline PEP & Pairwise error probability \\
\hline PSK & Phase shift keying \\
\hline QAM & Quadrature amplitude modulation \\
\hline SM & Spatial modulation \\
\hline $\mathrm{SE}$ & Spectral efficiency \\
\hline SNR & Signal-to-noise ratio \\
\hline SIC & Successive interference cancellation \\
\hline SWIPT & Simultaneous wireless information and power transfer \\
\hline
\end{tabular}

reconstructed OFDM-IM signal with the information of the cell-edge user is retransmitted.

2) On the basis of GCIM-NOMA as described above, we propose a CDM-NOMA scheme to increase the transmission data and further eliminate the IUI and SIC. In CDMNOMA, we employ different modulation modes (Mode I and Mode II) to clearly distinguish the subcarriers belonging to the cell-center and cell-edge users, respectively, and encode the incoming bit stream from these two users. On one hand, similar to DM-OFDM-IM, IM bits arise from the permutation of Mode I and Mode II (alternatively, from indices of the modulated subcarriers by Mode I or II). On the other hand, the users information will no longer overlap on the power domain, and thus reduce the interference and the complex SIC.

3) We analyze the bit error rate (BER) of GCIM-NOMA and CDM-NOMA over Rayleigh fading channels, where the optimal/suboptimal maximum-likelihood (ML) detection are applied to the cell-edge user. Moreover, tight upper bounds on the BER are derived in closed-form for the cell-center and cell-edge users. Furthermore, Monte Carlo simulations are carried out to verify the theoretical analysis and evaluate the BER performance, whose results show that the two proposed schemes have the potential to outperform C-NOMA in terms of BER as well as SE.

The remainder of this paper is organized as follows. Section II describes the basic system models and the optimal/suboptimal ML detectors for GCIM-NOMA and CDMNOMA. In Section III, closed-form expressions of the average BERs for these two proposed schemes are derived. The computer simulation results are detailed in Section IV, and finally, Section V concludes the paper. 
TABLE II

SUMMARY OF FrEQUENTLY USED SyMbols

\begin{tabular}{|c|c|}
\hline Symbol & Description \\
\hline$\rho$ & SNR \\
\hline$w$ & AWGN symbol \\
\hline$N_{0}$ & Noise variance \\
\hline $\mathbf{h}$ & Channel coefficients vector \\
\hline $\mathbf{x}$ & Transmitted vector \\
\hline$\lambda$ & Variance of the channel gain \\
\hline$I$ & Indices of selected subcarriers \\
\hline $\mathbf{s}$ & PSK/QAM symbol vector \\
\hline$\alpha$ & Power allocation factor \\
\hline$M_{A}\left(M_{B}\right)$ & Modulation order of user A (B) \\
\hline $\mathcal{S}_{A}\left(\mathcal{S}_{B}\right)$ & Constellation sets of cardinality $M_{A}\left(M_{B}\right)$ \\
\hline $\mathcal{X}$ & Superposed signal constellation alphabet \\
\hline
\end{tabular}

Notation: Scalar variables are in italic letters. Column vectors are denoted by lowercase bold letters. Superscripts ${ }^{T}$ and ${ }^{H}$ stand for transpose and Hermitian transpose, respectively. $\mathcal{C N}\left(0, \sigma^{2}\right)$ represents the complex Gaussian distribution with zero mean and variance $\sigma^{2} . E\{\cdot\}$ denotes the expectation operation. $C(\cdot, \cdot), Q(\cdot),\lfloor\cdot\rfloor$, and $|\cdot|$ are the binomial coefficient, the Gaussian $Q$-function, the floor function, and the absolute value operation, respectively. The probability of a random event is denoted by $\operatorname{Pr}(\cdot)$. The acronyms and frequently used symbols are listed in Tables I and II, respectively.

\section{SYSTEM MODEL}

As shown in Fig. 1, we consider a downlink NOMA scenario based on the OFDM framework, which consists of one BS (S) and two users (cell-center user A and cell-edge user B). Meanwhile, given the similarity between GCIM-NOMA and CDM-NOMA, Fig. 1 involves system models of the two proposed schemes. For brevity, we assume that all nodes are configured with a single transmit/receive antenna and operate in a half-duplex mode. Moreover, we define the channel coefficients of links $\mathrm{S} \rightarrow \mathrm{A}, \mathrm{S} \rightarrow \mathrm{B}$, and $\mathrm{A} \rightarrow \mathrm{B}$ as $\mathbf{h}_{A}, \mathbf{h}_{B_{1}}$, and $\mathbf{h}_{B_{2}}$, whose entries are modeled as independent and identically distributed complex Guassian random variables with the distribution $\mathcal{C N}\left(0, \lambda_{\mathrm{SA}}\right), \mathcal{C N}\left(0, \lambda_{\mathrm{SB}}\right)$ and $\mathcal{C N}\left(0, \lambda_{\mathrm{AB}}\right)$, respectively. According to the conclusion obtained in [25] that the variance of the channel gain is inversely proportional to the communication distance, we thereby have $\lambda_{\mathrm{SA}}>\lambda_{\mathrm{SB}}$. In the sequel, we further illustrate the complete communication protocol with two phases (broadcast phase and cooperative phase).

\section{A. Broadcast Phase}

At the beginning of this phase, users information processed by the IM-based OFDM is broadcast to users $\mathrm{A}$ and $\mathrm{B}$ at the same frequency and time slot via an $N \times 1$ OFDM block. We set the total number of the conveyed information bits as $m$. As for the construction of the transmitted symbol, this OFDM block is first divided into $g$ subblocks [18], each of which contains $p=m / g$ bits and independently performs the IM operation within $n=N / g$ subcarriers. Considering that the process for each subblock is completely independent and identical, we select the $\beta$-th subblock as an example to conduct the follow-up analysis, where $\beta \in\{1,2, \ldots, g\}$. We define

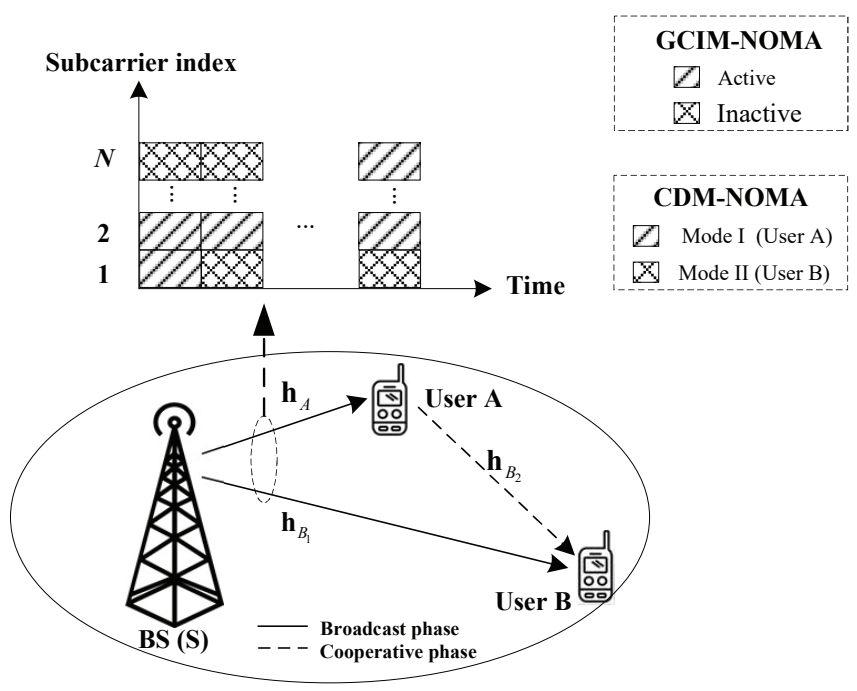

Fig. 1. Schematic diagram of GCIM-NOMA and CDM-NOMA.

$p=p_{1}+p_{2}$, where $p_{1}$ bits represent the additional information bits conveyed by the indices of active subcarriers, whereas $p_{2}$ bits are mapped onto the conventional constellation symbols. Notice that the $p_{1}$ input bits are assigned to select $k$ active subcarriers out of $n$ available ones to realize the corresponding IM procedures, i.e., $p_{1}=\left\lfloor\log _{2}(C(n, k))\right\rfloor$. The indices of the activated subcarriers are expressed as

$$
I^{(\beta)}=\left\{i^{(\beta)}(1), i^{(\beta)}(2), \ldots, i^{(\beta)}(k)\right\},
$$

where $i^{(\beta)}(\gamma) \in\{1,2, \ldots, n\}$ for $\gamma=1,2, \ldots, k$. Moreover, based on the above-mentioned different IM techniques, i.e., OFDM-IM and DM-OFDM-IM, we propose two methods to design and allocate $p_{2}$ information bits, corresponding to GCIM-NOMA and CDM-NOMA, respectively. For clarity, we first partition $p_{2}$ information bits into two parts, $p_{2}^{A}$ and $p_{2}^{B}$, to serve users A and B, respectively. Next, we further analyze two assignment criteria for $p_{2}$ bits, as detailed below.

1) GCIM-NOMA: Notice that in GCIM-NOMA, we only utilize the $k$ selected subcarriers to convey these $p_{2}$ information bits carried by the signal constellation, and we also apply the basic NOMA principle to generate the transmitted symbol on each active subcarrier. In general, to sustain a more reliable system, we tend to select a high modulation order $\left(M_{A}\right)$ in case of a better channel quality, while a low modulation level $\left(M_{B}\right)$ is used for a poor channel quality, i.e., $M_{A} \geq M_{B}$. Consequently, we have $p_{2}^{A}=k \log _{2}\left(M_{A}\right)$ and $p_{2}^{B}=k \log _{2}\left(M_{B}\right)$. Furthermore, we can write the phase shift keying (PSK)/quadrature amplitude modulation (QAM) constellation vector carrying $p_{2}$ bits as

$$
\mathbf{s}^{(\beta)}=\left[s^{(\beta)}(1), s^{(\beta)}(2), \ldots, s^{(\beta)}(k)\right]^{T} .
$$

It is worth noting that $s^{(\beta)}(\gamma)$ with $\gamma=1,2, \ldots, k$ is the superposition coded signal, expressed as

$$
s^{(\beta)}(\gamma)=\sqrt{\alpha} s_{A}^{(\beta)}(\gamma)+\sqrt{1-\alpha} s_{B}^{(\beta)}(\gamma),
$$

where $\alpha$ is the power allocation coefficient; $s_{A}^{(\beta)}(\gamma) \in \mathcal{S}_{A}$ and $s_{B}^{(\beta)}(\gamma) \in \mathcal{S}_{B}$ denote the modulated symbols of users A and B, 
respectively; $\mathcal{S}_{A}$ and $\mathcal{S}_{B}$ represent the complex PSK/QAM constellation sets of cardinality $M_{A}$ and $M_{B}$, respectively. Additionally, we have $s^{(\beta)}(\gamma) \in \mathcal{X}$, where $\mathcal{X}$ is a superposed signal constellation alphabet of size $M_{A} M_{B}$.

2) CDM-NOMA: As an extension of GCIM-NOMA, CDMNOMA is proposed on the basis of the following two reasons: one is that constrained by some idle subcarriers, the transmission rate always exists a bottleneck for GCIM-NOMA; the other one is that in order to further meet higher SE, the rise of $M_{A}$ and $M_{B}$ in GCIM-NOMA makes the interference among users more serious. Therefore, we consider a CDMNOMA scheme as illustrated in Fig. 1, in which the concept of dual-mode constellation modulation proposed in [21] is applied to increase the data rate by using all subcarriers. In Fig. 1, it is clear that $k$ subcarriers are assigned to user $\mathrm{B}$ and the remaining subcarriers $(n-k)$ are allocated to user A. Generally, we set $n-k>k$. To distinguish the subcarriers of users A and B clearly, we employ different modulation modes (Mode I and Mode II) to encode the incoming bit stream for users A and B, respectively. For CDM-NOMA with PSK modulation, Mode I and Mode II can be generated by the rotation of a basic $M$-ary PSK constellation [26], i.e., $M_{A}=M_{B}=M$; while for CDM-NOMA with QAM modulation, linear transformation should be performed on the basic $M$-ary QAM constellation to form multiple modes as described in [27]. Therefore, we have $p_{2}^{A}=k \log _{2}(M)$ and $p_{2}^{B}=(n-k) \log _{2}(M)$. Furthermore, we can also write the PSK/QAM constellation vector carrying $p_{2}$ bits as

$$
\mathbf{s}^{(\beta)}=\left[s^{(\beta)}(1), s^{(\beta)}(2), \ldots, s^{(\beta)}(k), \ldots, s^{(\beta)}(n)\right]^{T},
$$

where $s^{(\beta)}(\gamma)$ with $\gamma=1,2, \ldots, n$ can be expressed as

$$
s^{(\beta)}(\gamma)=\left\{\begin{array}{c}
s_{A}^{(\beta)}(\gamma), \gamma \notin I^{(\beta)} \\
s_{B}^{(\beta)}(\gamma), \gamma \in I^{(\beta)}
\end{array} .\right.
$$

Here, $s_{A}^{(\beta)}(\gamma) \in \mathcal{S}_{A}$ and $s_{B}^{(\beta)}(\gamma) \in \mathcal{S}_{B}$ denote the modulated symbols of users A and B, respectively; $\mathcal{S}_{A}$ and $\mathcal{S}_{B}$ represent the Mode I and Mode II constellation sets of cardinality $M$, respectively. At this point, the allocation of $p_{2}$ message bits has been carried out.

Notice that the IM bits $p_{1}$ transmitted by activating $k$ out of $n$ subcarriers are allocated to serve user B both in GCIMNOMA and CDM-NOMA. This can be explained by three reasons: the first reason is that the $\mathrm{SE}$ of user $\mathrm{B}$ is smaller than that of user A in these schemes; the second reason is that the channel quality of $\mathrm{S} \rightarrow \mathrm{B}$ is rather poor, compared with that of $\mathrm{S} \rightarrow \mathrm{A}$; the last one is that by contrast with the conventional modulation bits, the transmission of IM bits is near error-free at the high signal-to-ratio (SNR) region and and can further improve the performance of weak users. Therefore, we can obtain $p_{A}=p_{2}^{A}$ and $p_{B}=p_{1}+p_{2}^{B}$. After obtaining $I^{(\beta)}$ and $\mathbf{s}^{(\beta)}$, the transmitted vector for the $\beta$-th subblock is created as

$$
\begin{aligned}
\mathbf{x}^{(\beta)} & =\left[x^{(\beta)}(1), \ldots, x^{(\beta)}(n)\right]^{T} \\
& =[x((\beta-1) n+1), \ldots, x(\beta n)]^{T},
\end{aligned}
$$

where $x^{(\beta)}(\gamma) \in\{0, \mathcal{X}\}$ with $\gamma=1,2, \ldots, n$ for GCIMNOMA, and $x^{(\beta)}(\gamma) \in\left\{\mathcal{S}_{A}, \mathcal{S}_{B}\right\}$ with $\gamma=1,2, \ldots, n$ for CDM-NOMA. All OFDM subblocks are then concatenated together to generate an OFDM block. Afterwards, the remaining procedures to be implemented are the same as those of classical OFDM, which mainly include the inverse fast Fourier transform, cyclic prefix (CP) appending, and parallel-to-series conversion. When the above processes are completed, the transmitted symbol at the BS can be successfully broadcast to users $\mathrm{A}$ and $\mathrm{B}$.

At the receiving side, the fast Fourier transform is applied after removing the $\mathrm{CP}$. Therefore, the frequency-domain received signals of the $\beta$-th subblock at users $\mathrm{A}$ and $\mathrm{B}$ are given by

$$
y_{A}^{(\beta)}(\gamma)=h_{A}^{(\beta)}(\gamma) x^{(\beta)}(\gamma)+w_{A}^{(\beta)}(\gamma),
$$

and

$$
y_{B_{1}}^{(\beta)}(\gamma)=h_{B_{1}}^{(\beta)}(\gamma) x^{(\beta)}(\gamma)+w_{B_{1}}^{(\beta)}(\gamma),
$$

respectively, where $\gamma=1,2, \ldots, n ; h_{A}^{(\beta)}(\gamma)$ and $h_{B_{1}}^{(\beta)}(\gamma)$ are the $((\beta-1) n+\gamma)$-th elements of $\mathbf{h}_{A}$ and $\mathbf{h}_{B_{1}}$, respectively; $w_{A}^{(\beta)}(\gamma)$ and $w_{B_{1}}^{(\beta)}(\gamma)$ are the additive white Gaussian noise (AWGN) variables for links $\mathrm{S} \rightarrow \mathrm{A}$ and $\mathrm{S} \rightarrow \mathrm{B}$, respectively, both following the distribution $\mathcal{C N}\left(0, N_{0}\right)$. For GCIM-NOMA, we define the transmit SNR per subcarrier as $\rho_{S A}=\rho_{S B}=k /\left(n N_{0}\right)$ in the broadcast phase, whereas for CDM-NOMA, the transmit SNR per subcarrier is defined as $\rho_{S A}=\rho_{S B}=1 / N_{0}$. User B retains the received signal $y_{B_{1}}^{(\beta)}(\gamma)$ with $\beta=1,2, \ldots, g$ and $\gamma=1,2, \ldots, n$ for demodulation in the follow-up phase, whereas user A recovers the transmitted signals, including the transmitted symbol of user B (alternatively, $I^{(\beta)}$ and $\mathbf{s}_{B}^{(\beta)}$ ) and its own signal (alternatively, $\mathbf{s}_{A}^{(\beta)}$ ). Assuming that the well-known ML detector algorithm is applied, the detection process without SIC can be formulated as

$$
\left(\hat{I}^{(\beta)}, \hat{\mathbf{s}}^{(\beta)}\right)=\underset{I^{(\beta)}, \mathbf{s}^{(\beta)}}{\arg \min } \sum_{\gamma=1}^{n}\left|y_{A}^{(\beta)}(\gamma)-h_{A}^{(\beta)}(\gamma) x^{(\beta)}(\gamma)\right|^{2},
$$

where $\hat{I}^{(\beta)}\left(\hat{\mathbf{s}}^{(\beta)}\right)$ is the estimate of $I^{(\beta)}\left(\mathbf{s}^{(\beta)}\right)$. From (9), it can be easily found that the ML detector considers all possible realizations for the $\beta$-th subblock by searching all possible subcarrier index combinations and the signal constellations, whose computational complexity is $\mathcal{O}\left(2^{\left\lfloor\log _{2}(C(n, k))\right\rfloor+p_{2}}\right)$. Finally, user A further recovers its own information bits from $\hat{\mathbf{s}}_{A}^{(\beta)}$ and then preserves $\hat{I}^{(\beta)}$ and $\hat{\mathbf{s}}_{B}^{(\beta)}$ for cooperative transmission in the next phase.

\section{B. Cooperative Phase}

With the estimated symbols of user B, i.e., $\hat{I}^{(\beta)}$ and $\hat{\mathbf{s}}_{B}^{(\beta)}$, a new OFDM-IM signal $\hat{\mathbf{x}}_{B}^{(\beta)}$ can be regenerated following an identical procedure to (6). Next, user A forwards $\hat{\mathbf{x}}_{B}^{(\beta)}$ to user B with full transmit power, and the received signal at user B can be represented by

$y_{B_{2}}^{(\beta)}(\gamma)=h_{B_{2}}^{(\beta)}(\gamma) \hat{x}_{B}^{(\beta)}(\gamma)+w_{B_{2}}^{(\beta)}(\gamma), \gamma=1,2, \ldots, n$,

where $h_{B_{2}}^{(\beta)}(\gamma)$ is the $((\beta-1) n+\gamma)$-th column of $\mathbf{h}_{B_{2}}$ and $w_{B_{2}}^{(\beta)}(\gamma)$ is the AWGN symbol at user $\mathbf{B}$, distributed as 
$\mathcal{C N}\left(0, N_{0}\right)$. Notice that the SNR should be expressed as $\rho_{A B}=k /\left(n N_{0}\right)$ for all proposed schemes. Due to the similarity of the detection algorithms for the two proposed schemes in the cooperative phase, we mainly focus on GCIM-NOMA to describe the detection process for user B. Additionally, considering the higher computational complexity at user $\mathrm{B}$, we design and implement two different detectors for user B, i.e., the optimal ML detector and the low-complexity sub-optimal ML detector.

1) Optimal ML Detector: To achieve better performance, the maximal ratio combining (MRC) is employed at user B, where $y_{B_{1}}^{(\beta)}(\gamma)$ in $(8)$ and $y_{B_{2}}^{(\beta)}(\gamma)$ in (10) with weights

$$
\varphi_{1}^{(\beta)}(\gamma)=\frac{\left(h_{B_{1}}^{(\beta)}(\gamma)\right)^{H} \sqrt{1-\alpha}}{\left|h_{B_{1}}^{(\beta)}(\gamma)\right|^{2} \alpha+N_{0}}, \varphi_{2}^{(\beta)}(\gamma)=\frac{\left(h_{B_{2}}^{(\beta)}(\gamma)\right)^{H}}{N_{0}}
$$

are combined together, forming the following signal,

$$
\begin{aligned}
y_{B}^{(\beta)}(\gamma)= & \varphi_{1}^{(\beta)}(\gamma) y_{B_{1}}^{(\beta)}(\gamma)+\varphi_{2}^{(\beta)}(\gamma) y_{B_{2}}^{(\beta)}(\gamma) \\
= & \varphi_{1}^{(\beta)}(\gamma) h_{B_{1}}^{(\beta)}(\gamma) x^{(\beta)}(\gamma)+\varphi_{2}^{(\beta)}(\gamma) h_{B_{2}}^{(\beta)}(\gamma) \hat{x}_{B}^{(\beta)}(\gamma) \\
& +\varphi_{1}^{(\beta)}(\gamma) w_{B_{1}}^{(\beta)}(\gamma)+\varphi_{2}^{(\beta)}(\gamma) w_{B_{2}}^{(\beta)}(\gamma) .
\end{aligned}
$$

Assuming that $\hat{\mathbf{x}}_{B}^{(\beta)}$ has been correctly detected at user A, user $B$ is capable of recovering its own signal by (12) based on the ML detection criterion without SIC below:

$$
\begin{aligned}
\left(\tilde{I}^{(\beta)}, \tilde{\mathbf{s}}_{B}^{(\beta)}\right)= & \underset{I^{(\beta)}, \mathbf{s}_{B}^{(\beta)}}{\arg \min } \sum_{\gamma=1}^{n} \mid y_{B}^{(\beta)}(\gamma)-x_{B}^{(\beta)}(\gamma) \\
& \times\left.\left(\varphi_{1}^{(\beta)}(\gamma) h_{B_{1}}^{(\beta)}(\gamma)+\varphi_{2}^{(\beta)}(\gamma) h_{B_{2}}^{(\beta)}(\gamma)\right)\right|^{2},
\end{aligned}
$$

where $x_{B}^{(\beta)}(\gamma)$ is $\gamma$-th variable of $\mathbf{x}_{B}^{(\beta)}$, and $\mathbf{x}_{B}^{(\beta)}$ is an OFDM signal generated by $I^{(\beta)}(\gamma)$ and $\mathbf{s}_{B}^{(\beta)}(\gamma)$. Obviously, the computational complexity of the optimal ML detector in terms of the number of complex multiplications is $\mathcal{O}\left(2^{\left\lfloor\log _{2}(C(n, k))\right\rfloor} M_{B}{ }^{k}\right)$.

2) Low-Complexity Suboptimal ML Detector: Different from the optimal ML detector, we can alternatively obtain the indices of the activated subcarriers $I^{(\beta)}$ by greedy detector (GD), i.e., selecting $k$ maximum combined energies,

$$
\tilde{I}^{(\beta)}=\underset{I^{(\beta)}}{\arg \max }\left\{\mathcal{Y}_{1}+\mathcal{Y}_{2}\right\},
$$

where we have

$$
\begin{aligned}
& \mathcal{Y}_{1}=\left|y_{B_{1}}^{(\beta)}\left(i^{(\beta)}(1)\right)\right|^{2}+\cdots\left|y_{B_{1}}^{(\beta)}\left(i^{(\beta)}(k)\right)\right|^{2}, \\
& \mathcal{Y}_{2}=\left|y_{B_{2}}^{(\beta)}\left(i^{(\beta)}(1)\right)\right|^{2}+\cdots\left|y_{B_{2}}^{(\beta)}\left(i^{(\beta)}(k)\right)\right|^{2} .
\end{aligned}
$$

Afterwards, the MRC is employed to combine the constellation signals, followed by the ML detector to recover these information bits, given by,

$$
\begin{aligned}
\tilde{\mathbf{s}}_{B}^{(\beta)}= & \underset{\mathbf{s}_{B}^{(\beta)}}{\arg \min } \sum_{\gamma \in \tilde{I}^{(\beta)}} \mid y_{B}^{(\beta)}(\gamma)-x_{B}^{(\beta)}(\gamma) \\
& \times\left.\left(\varphi_{1}^{(\beta)}(\gamma) h_{B_{1}}^{(\beta)}(\gamma)+\varphi_{2}^{(\beta)}(\gamma) h_{B_{2}}^{(\beta)}(\gamma)\right)\right|^{2} .
\end{aligned}
$$

From (13) and (16), it is clear that the computational complexity is $\mathcal{O}\left(2^{\left\lfloor\log _{2}(C(n, k))\right\rfloor}+M_{B}{ }^{k}\right)$, which is much lower than that of the ML detector.
Remark 1: Similar to GCIM-NOMA, we can also deign two different detectors, i.e., optimal/sub-optimal detectors, for CDM-NOMA. For the optimal ML detector, the MRC is first employed with the following weights:

$$
\phi_{1}^{(\beta)}(\gamma)=\frac{\left(h_{B_{1}}^{(\beta)}(\gamma)\right)^{H}}{N_{0}}, \phi_{2}^{(\beta)}(\gamma)=\frac{\left(h_{B_{2}}^{(\beta)}(\gamma)\right)^{H}}{N_{0}}
$$

to combine the different received signals, whose expression can be written as (12) with $\varphi_{1}^{(\beta)}(\gamma)$ and $\varphi_{2}^{(\beta)}(\gamma)$ replaced by $\phi_{1}^{(\beta)}(\gamma)$ and $\phi_{2}^{(\beta)}(\gamma)$, respectively. As illustrated in (13), a joint decision on the selected indices and the constellation symbols can be carried out to recover the message of user B. On the other side, the high computational complexity of order $\mathcal{O}\left(2^{\left\lfloor\log _{2}(C(n, k))\right\rfloor} M_{A}{ }^{n-k} M_{B}{ }^{k}\right)$ arising from this brute-force search cannot be neglected. Therefore, to effectively reduce the complexity, the low-complexity sub-optimal ML detector is then proposed and implemented in the following analysis. As described in (14) and (15), we first decode the index bits and obtain the active subcarriers (alternatively, the subcarriers allocated to user B) according to the information from the link $\mathrm{A} \rightarrow \mathrm{B}$, via

$$
\tilde{I}^{(\beta)}=\underset{I^{(\beta)}, \mathbf{s}_{B}^{(\beta)}}{\arg \min } \sum_{\gamma=1}^{n}\left|y_{B_{2}}^{(\beta)}(\gamma)-h_{B_{2}}^{(\beta)}(\gamma) x_{B}^{(\beta)}(\gamma)\right|^{2} .
$$

This approach is proposed based on the highly reliable transmission of IM bits. Then, the MRC is employed for the signal from links $\mathrm{S} \rightarrow \mathrm{B}$ and $\mathrm{A} \rightarrow \mathrm{B}$ to demodulate the symbol bits carried by the detected active subcarriers. As can be seen from Section IV, the suboptimal detector achieves near-optimal performance with greatly reduced computational complexity of order $\mathcal{O}\left(\left(2^{\left\lfloor\log _{2}(C(n, k))\right\rfloor}+1\right) M_{B}{ }^{k}\right)$.

\section{Performance Analysis}

In this section, we derive upper bounds on the BERs of GCIM-NOMA for users A and B, assuming perfect channel estimation and the optimal ML detector. Incidentally, the superscript $(\beta)$ is omitted for clarity in the subsequent analysis. Regarding the BER analysis of CDM-NOMA, we also provide a remark to highlight it since the analysis process overlaps with that of GCIM-NOMA greatly.

\section{A. BER of User A}

From (9), it is clear that the estimation error of $\mathbf{s}_{A}$ will arise from whether the detection for the index of active subcarrier or the SIC process is performed correctly.

For clarity, let us first study the index error probability (IEP). We define $\operatorname{Pr}(I \rightarrow \hat{I})$ as the probability of erroneously detecting $I$ as $\hat{I}$, which can be calculated by

$$
\operatorname{Pr}(I \rightarrow \hat{I})=\frac{1}{2^{p_{2}}} \sum_{\mathbf{s}, \hat{\mathbf{s}}} \operatorname{Pr}(\mathbf{x} \rightarrow \hat{\mathbf{x}} \mid \mathbf{s}, \hat{\mathbf{s}}, I, \hat{I}),
$$

where $\operatorname{Pr}(\mathbf{x} \rightarrow \hat{\mathbf{x}} \mid \mathbf{s}, \hat{\mathbf{s}}, I, \hat{I})$ represents the conditional pairwise error probability (PEP) of detecting $\mathbf{x}$ as $\hat{\mathbf{x}}$ conditioned on $\mathbf{s}, \hat{\mathbf{s}}$, 
$I$, and $\hat{I}$. This conditional PEP can be obtained via averaging the $\operatorname{Pr}\left(\mathbf{x} \rightarrow \hat{\mathbf{x}} \mid \mathbf{s}, \hat{\mathbf{s}}, I, \hat{I}, \mathbf{h}_{A}\right)$ over $\mathbf{h}_{A}$, represented by

$$
\begin{aligned}
\operatorname{Pr}(\mathbf{x} \rightarrow \hat{\mathbf{x}} \mid \mathbf{s}, \hat{\mathbf{s}}, I, \hat{I}) & \\
=E_{\mathbf{h}_{A}} & \left\{\operatorname{Pr}\left(\mathbf{x} \rightarrow \hat{\mathbf{x}} \mid \mathbf{s}, \hat{\mathbf{s}}, I, \hat{I}, \mathbf{h}_{A}\right)\right\} \\
=E_{\mathbf{h}_{A}} & \left\{\operatorname { P r } \left(\sum_{\gamma=1}^{n}\left|y_{A}(\gamma)-h_{A}(\gamma) x(\gamma)\right|^{2}\right.\right. \\
& \left.\left.>\sum_{\gamma=1}^{n}\left|y_{A}(\gamma)-h_{A}(\gamma) \hat{x}(\gamma)\right|^{2}\right)\right\} \\
=E_{\mathbf{h}_{A}} & \left\{Q\left(\sqrt{\frac{1}{2 N_{0}} \sum_{\gamma=1}^{n} G(\gamma)}\right)\right\},
\end{aligned}
$$

where $G(\gamma)=\left|h_{A}(\gamma)(\hat{x}(\gamma)-x(\gamma))\right|^{2}$. Additionally, we can approximate (20) quite well by employing [28]

$$
Q(x) \approx \frac{1}{12} e^{-x^{2} / 2}+\frac{1}{4} e^{-2 x^{2} / 3}
$$

Further, substituting the approximation of (20) into (19) yields

$$
\begin{aligned}
\operatorname{Pr}(I \rightarrow \hat{I})=\frac{1}{2^{p_{2}}} \sum_{\mathbf{s}, \hat{\mathbf{s}}} & \left\{\frac{1}{12} \prod_{\gamma=1}^{n} E_{\mathbf{h}_{A}}\left(e^{-q_{1} G(\gamma)}\right)\right. \\
+ & \left.\frac{1}{4} \prod_{\gamma=1}^{n} E_{\mathbf{h}_{A}}\left(e^{-q_{2} G(\gamma)}\right)\right\},
\end{aligned}
$$

where $q_{1}=1 /\left(4 N_{0}\right)$ and $q_{2}=1 /\left(3 N_{0}\right)$. Considering that the moment generating function (MGF) of $\left|h_{A}(\gamma)\right|^{2}$ is

$$
\mathcal{M}(t)=\frac{1}{1-2 t \lambda_{S A}},
$$

we have

$$
E_{\mathbf{h}_{A}}\left(e^{-q_{i} G(\gamma)}\right)=\frac{1}{1-2 q_{i} \lambda_{S A}|x(\gamma)-\hat{x}(\gamma)|^{2}},
$$

where $i=1,2$ and $\gamma=1,2, \ldots, n$. By substituting (24) into (22), we can obtain the final expression of $\operatorname{Pr}(I \rightarrow \hat{I})$.

Furthermore, it is essential to calculate the error probability of the SIC process, while the IEP has already been obtained. We first acknowledge that due to the specific characteristics of superposed signal constellation, there is no general formula to describe the error probability of the SIC process at present. Let us take $M_{A}=4$ and $M_{B}=2$ as an example to perform the following analysis. Meanwhile, the corresponding error probability of SIC has already been derived in [29], represented by

$$
P_{S I C}=\frac{1}{4}\left[\left(1-\sqrt{\frac{\bar{\rho}_{1}}{2+\bar{\rho}_{1}}}\right)+\left(1-\sqrt{\frac{\bar{\rho}_{2}}{2+\bar{\rho}_{2}}}\right)\right],
$$

where $\bar{\rho}_{1}$ and $\bar{\rho}_{2}$ are defined as

$$
\bar{\rho}_{1}=\frac{n \lambda_{S A}}{k} \frac{(\sqrt{\alpha}+\sqrt{2(1-\alpha)})^{2}}{N_{0}},
$$

and

$$
\bar{\rho}_{2}=\frac{n \lambda_{S A}}{k} \frac{(\sqrt{\alpha}-\sqrt{2(1-\alpha)})^{2}}{N_{0}} .
$$

With the aid of (22) and (25), we can derive the bit error probability (BEP) contributed by the error event that the indices of activated subcarriers are detected incorrectly, expressed as

$$
\begin{aligned}
P_{A_{1}} \leq & \frac{1}{2^{p_{1}}} \sum_{I} \sum_{\hat{I}, I \neq \hat{I}} \operatorname{Pr}(I \rightarrow \hat{I})\left[\frac{1}{2} \bar{D}(I, \hat{I})\right. \\
& \left.+\left\{P_{A b}\left(M_{A}\right)\left(1-P_{S I C}\right)+\frac{1}{2} P_{S I C}\right\} D(I, \hat{I})\right],
\end{aligned}
$$

where $D(I, \hat{I})$ and $\bar{D}(I, \hat{I})$ denote the numbers of the activated subcarriers detected correctly or incorrectly, respectively. $P_{A b}\left(M_{A}\right)$ is the average BEP of $M_{A}$-PSK over a Rayleigh fading channel [30], represented by

$$
\begin{aligned}
P_{A b}\left(M_{A}\right) & =\left(\frac{M_{A}-1}{M_{A}}\right)\left\{1-\sqrt{\frac{g_{\mathrm{PSK}} \rho_{b}}{1+g_{\mathrm{PSK}} \rho_{b}}}\left(\frac{M_{A}}{\left(M_{A}-1\right) \pi}\right)\right. \\
& \left.\times\left[\frac{\pi}{2}+\tan ^{-1}\left(\sqrt{\frac{g_{\mathrm{PSK}} \rho_{b}}{1+g_{\mathrm{PSK}} \rho_{b}}} \cot \left(\frac{\pi}{M_{A}}\right)\right)\right]\right\},
\end{aligned}
$$

where $\rho_{b}=\alpha \lambda_{A B} \rho_{S A} / \log _{2}\left(M_{A}\right)$ and $g_{\mathrm{PSK}} \triangleq \sin ^{2} \pi / M_{A}$. Correspondingly, the expression of $P_{A b}$ for $M_{A}$-QAM can also be found from [30]. A detailed description of the derivations for (28) is presented in Appendix A.

When the indices of activated subcarriers $I$ are detected perfectly, the BEP of user A can be given by

$$
P_{A_{2}} \approx\left(1-P_{I A}\right)\left(P_{A b}\left(M_{A}\right)\left(1-P_{S I C}\right)+\frac{1}{2} P_{S I C}\right) .
$$

As for the IEP $P_{I A}$, according to (22), we have

$$
P_{I A}=\frac{1}{2^{p_{1}}} \sum_{I} \sum_{\hat{I}, I \neq \hat{I}} \operatorname{Pr}(I \rightarrow \hat{I}) .
$$

The overall BER upper bound of user A can be calculated by adding (28) and (31) together, i.e.,

$$
P_{A} \leq P_{A_{1}}+P_{A_{2}} .
$$

Finally, it is clear from (32) that the diversity order of user A is determined by $P_{A b}\left(M_{A}\right)$, which yields unity.

\section{B. BER of User B}

In this subsection, we derive the BER upper bound for user B. Considering the following two facts: 1) the near errorfree transmission of IM bits at the high SNR region; and 2) the better channel quality of the link $\mathrm{A} \rightarrow \mathrm{B}$, we will mainly use the signal received from the link $\mathrm{A} \rightarrow \mathrm{B}$ to calculate the IEP, and then employ the combined information in (16) to obtain the BEP contributed by the constellation signal. Thus, we can obtain the BER expression of user B when different error estimations are considered, expressed as (33), shown on the top of this page, where

$$
\eta_{1}=\frac{p_{2}^{B}}{p_{1}+p_{2}^{B}}, \eta_{2}=\frac{p_{1}+\frac{1}{2} p_{2}^{B}}{p_{1}+p_{2}^{B}} .
$$




$$
\tilde{P}_{B}=\left\{\begin{array}{l}
\frac{1}{2} P_{I A}, \text { if }\{\hat{I} \neq I\} \\
P_{B b} \eta_{1}\left(1-P_{I A}\right)\left(1-P_{S I C}\right) \bar{P}_{I B_{1}}, \text { if }\left\{\hat{I}, \tilde{I}=I, \hat{\mathbf{s}}_{B}=\mathbf{s}_{B}\right\} \\
\frac{1}{2} \eta_{1}\left(1-P_{I A}\right) P_{S I C} \bar{P}_{I B_{2}}, \text { if }\left\{\hat{I}, \tilde{I}=I, \hat{\mathbf{s}}_{B} \neq \mathbf{s}_{B}\right\} \\
\eta_{2}\left(1-P_{I A}\right)\left(1-P_{S I C}\right) P_{I B_{1}}, \text { if }\left\{\hat{I}=I \neq \tilde{I}, \hat{\mathbf{s}}_{B}=\mathbf{s}_{B}\right\} \\
\eta_{2}\left(1-P_{I A}\right) P_{S I C} P_{I B_{2}}, \text { if }\left\{\hat{I}=I \neq \tilde{I}, \hat{\mathbf{s}}_{B} \neq \mathbf{s}_{B}\right\}
\end{array}\right.
$$

In (33), $\hat{I}, \tilde{I}$, and $\hat{\mathbf{s}}_{B}$ have been defined in Section II. $P_{B b}$ is the average BER of $M_{B}$-PSK/QAM over Rayleigh fading channels after MRC. For the PSK scenario, $P_{B b}$ is given by [31]

$P_{B b}=\left\{\begin{array}{l}\left(\frac{1-\mu}{2}\right)^{L} \sum_{l=0}^{L-1}\left(\begin{array}{c}L-1+l \\ l\end{array}\right)\left(\frac{1+\mu}{2}\right)^{l}, \quad M_{B}=2 \\ \frac{1}{2}\left[1-\frac{\mu}{\sqrt{2-\mu^{2}}} \sum_{l=0}^{L-1}\left(\begin{array}{c}2 l \\ l\end{array}\right)\left(\frac{1-\mu^{2}}{4-2 \mu^{2}}\right)^{l}\right], M_{B}=4\end{array}\right.$

where $\mu=\sqrt{\frac{\bar{\rho}}{1+\bar{\rho}}}, \bar{\rho}=\frac{1}{2}\left\{\lambda_{A B} \rho_{A B}+\frac{\lambda_{A B} \rho_{S B}(1-\alpha)}{\lambda_{A B} \rho_{S B} \alpha+1}\right\}$, and $L=2$. Correspondingly, $P_{B b}$ for QAM modulation can also be found from [31]. Additionally, we assume conservatively that when an error in detecting the active subcarrier index occurs at user A or B, the BER from the constellation signal is approximately as $1 / 2 . P_{S I C}$ and $P_{I A}$ have been computed in (25) and (31). $P_{I B_{1}}$ and $P_{I B_{2}}$ stand for the IEP at user B, when both $I$ and $\mathbf{s}_{B}$ are decoded correctly or $I$ is detected correctly but $\mathbf{s}_{B}$ cannot be decoded correctly at user A, respectively. By contrast, $\bar{P}_{I B_{1}}$ and $\bar{P}_{I B_{2}}$ denote the corresponding probability of correct detection. By using the similar method for obtaining (22) and (31), we can calculate $P_{I B_{1}}$ as

$$
\begin{aligned}
P_{I B_{1}}= & \frac{1}{2^{p}} \sum_{I, \hat{I}} \sum_{\mathbf{x}, \hat{\mathbf{x}}}\left\{\frac{1}{12} \prod_{\gamma=1}^{n} E_{\mathbf{h}_{B_{2}}}\left(e^{-q_{1} G_{2}(\gamma)}\right)\right. \\
+ & \left.\frac{1}{4} \prod_{\gamma=1}^{n} E_{\mathbf{h}_{B_{2}}}\left(e^{-q_{2} G_{2}(\gamma)}\right)\right\},
\end{aligned}
$$

where $G_{2}(\gamma)=\left|h_{B_{2}}(\gamma)\left(x_{B}(\gamma)-\tilde{x}_{B}(\gamma)\right)\right|^{2}$ and $\tilde{x}_{B}(\gamma)$ is estimate of $x_{B}(\gamma)$ in the cooperative phase. Hence, we also have

$$
\bar{P}_{I B_{1}}=1-P_{I B_{1}} .
$$

Regarding $P_{I B_{2}}$ and $\bar{P}_{I B_{2}}$, given that $\mathbf{s}_{B}$ has been detected incorrectly at user $\mathrm{A}$, we will calculate their upper bound by

$$
\bar{P}_{I B_{2}} \leq 1-P_{I}^{A B} P_{I}^{S B}, n>2,
$$

and

$$
P_{I B_{2}} \leq 1-\left(1-P_{I}^{A B}\right)\left(1-P_{I}^{S B}\right), n>2,
$$

where $P_{I}^{A B}$ and $P_{I}^{S B}$ denote the IEPs pertaining to links $\mathrm{S} \rightarrow \mathrm{B}$ and $\mathrm{A} \rightarrow \mathrm{B}$, respectively. For $n=2, \bar{P}_{I B_{1}}=P_{I B_{1}}=1 / 2$. For brevity, we can employ the GD detector to recover the index bits at user B [32]. Considering that the GD detector uses the $k$ maximum received energies as a test statistic, we define that an active subcarrier index is incorrectly detected when the maximum noise energy of any inactive subcarrier $(\bar{\gamma})$ is greater than the energy of an active one $(\gamma)$, i.e., $(\gamma \rightarrow \bar{\gamma})$. Hence, we can write the error probability of $(\gamma \rightarrow \bar{\gamma})$ as [32]

$$
\operatorname{Pr}(\gamma \rightarrow \bar{\gamma})=1-\sum_{i=0}^{n-k} C(n-k, i) \frac{-1^{i}}{i+1} e^{\hat{\rho}\left(\frac{1}{i+1}-1\right)},
$$

where $\hat{\rho}$ is the SNR of each link. With the law of total probability, the overall IEP can be expressed as

$$
\begin{aligned}
P_{I} & =\sum_{\gamma=1}^{n} \operatorname{Pr}(\gamma \rightarrow \bar{\gamma}) P(\gamma) \\
& =k\left\{1-\sum_{i=0}^{n-k} C(n-k, i) \frac{-1^{i}}{i+1}\left(\frac{i+1}{i(\hat{\rho}+1)+1}\right)\right\},
\end{aligned}
$$

where $P(\gamma)=k / n$. Therefore, we can obtain the expressions of $P_{I}^{A B}$ and $P_{I}^{S B}$ by using (41). Substituting the above results into (33), $\tilde{P}_{B}$ can be calculated successfully. Further, the overall BER upper bound of user B can be determined in closed-form by adding up all possible $\tilde{P}_{B}$. Finally, it is clear from (33) that user B can achieve a diversity order of unity, which is determined by $P_{S I C}$ and $P_{B b}$.

Remark 2: Following the BER analysis of GCIM-NOMA, we also provide the final BER expressions for users A and B in the CDM-NOMA scheme. For user A, an error occurs when subcarriers are misclassified or the transmitted symbol is detected incorrectly. According to (28), the BER upper bound of user A can be written as

$$
P_{A} \leq \frac{1}{2^{p_{1}}} \sum_{I, \hat{I}} \operatorname{Pr}(I \rightarrow \hat{I})\left[D(I, \hat{I}) P_{A b}(M)+\frac{1}{2} \bar{D}(I, \hat{I})\right] .
$$

For user $\mathrm{B}$, according to the BER analysis previously described in GCIM-NOMA, we can find the bit errors of user B also arise from different cases, which is illustrated in details in (33). Therefore, we can derive the BER upper bound of user $\mathrm{B}$ as

$$
\begin{aligned}
P_{B} & \leq \frac{1}{2} P_{I A}+\left(1-P_{I A}\right) P_{A b}(M)\left\{\eta_{1}\left(1-P_{I B_{2}}\right)+P_{I B_{2}}\right\} \\
& +\left(1-P_{I A}\right)\left(1-P_{A b}(M)\right)\left\{P_{B b} \eta_{1}\left(1-P_{I B_{1}}\right)+\eta_{2} P_{I B_{1}}\right\} .
\end{aligned}
$$

Different from GCIM-NOMA, in (43), we have $P_{I B_{2}}=P_{I}^{A B}$. This is because that the information from the link $\mathrm{S} \rightarrow \mathrm{B}$ cannot be employed to recover the index bits by the GD detector. It is assumed that the error probability of $\mathbf{s}_{B}$ is one when $\mathbf{s}_{B}$ has been decoded incorrectly at user A. Further, other parameters have been explicitly defined and computed in Section III-B, but still need to be adjusted correspondingly. Finally, similar to GCIM-NOMA, we can observe from (42) 


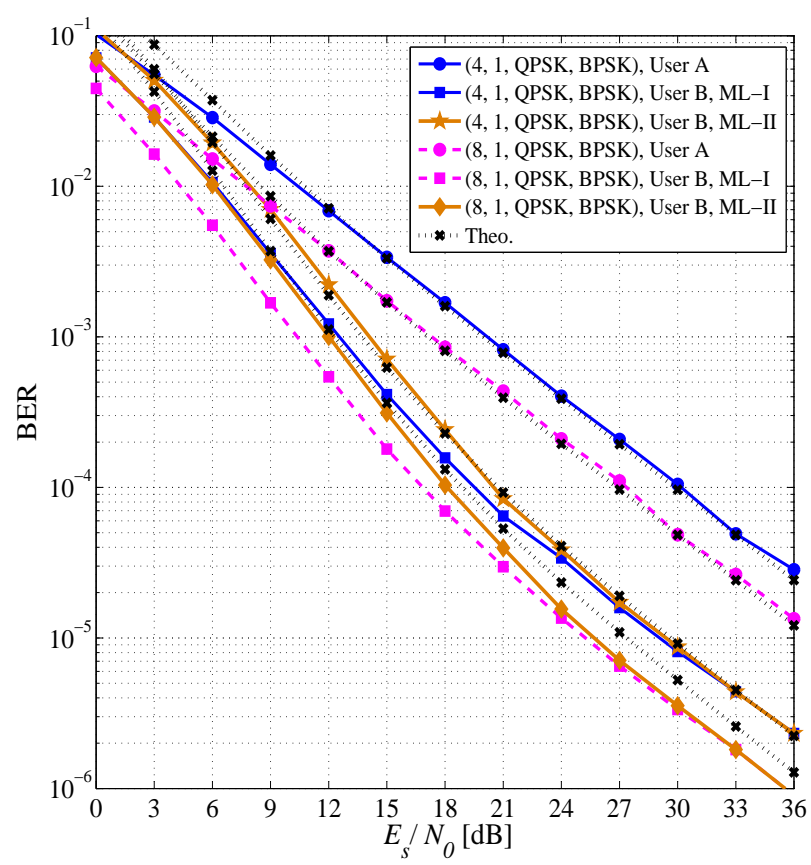

Fig. 2. BER versus $E_{s} / N_{0}$ performance of the GCIM-NOMA scheme for numerical verification purposes: different numbers of subcarriers $n$.

and (43) that users A and B both can achieve a diversity of unity, which is mainly determined by $P_{A b}(M)$.

\section{Simulation Results and Discussion}

In this section, we employ Monte Carlo simulations to demonstrate the uncoded BER performance of the proposed GCIM-NOMA and CDM-NOMA systems, and include CNOMA investigated in [13] as a benchmark to evaluate the system performance. Notice that in C-NOMA, user B employs the MRC to jointly combine the received signals. First, we compare the theoretical results with their simulation counterparts to verify the effectiveness of the derived BER expressions, and then we also investigate the impact of different numbers of subcarriers on the performance of the proposed schemes. Moreover, the achievable BER performances of GCIM-NOMA and CDM-NOMA are compared based on simulation results. Further BER comparisons are performed between these proposed schemes and the C-NOMA.

Note that in all simulations, we assume that the channels experience independent Rayleigh fading, and the channel coefficients are perfectly known to users A and B [13]. Meanwhile, in the considered simulations, the SNR is defined as $E_{s} / N_{0}$, except in Fig. 7 where the SNR is set to be $E_{b} / N_{0}$. Here, $E_{s}$ and $E_{b}$ denote the average symbol energy and average bit energy, respectively. We also set $\lambda_{S B}=1, \lambda_{S A}=6$, $\lambda_{A B}=2$. For brevity, we will refer to "GCIM-NOMA ( $n$, $k, M_{A}$-PSK, $M_{B}$-PSK)" as the GCIM-NOMA scheme, in which only $k$ subcarriers out of $n$ available ones per group are activated to convey the superposition coded symbols from (3) at the BS, "CDM-NOMA ( $n, k, M$-PSK)" as the CDMNOMA scheme, in which $k$ subcarriers are assigned to user B

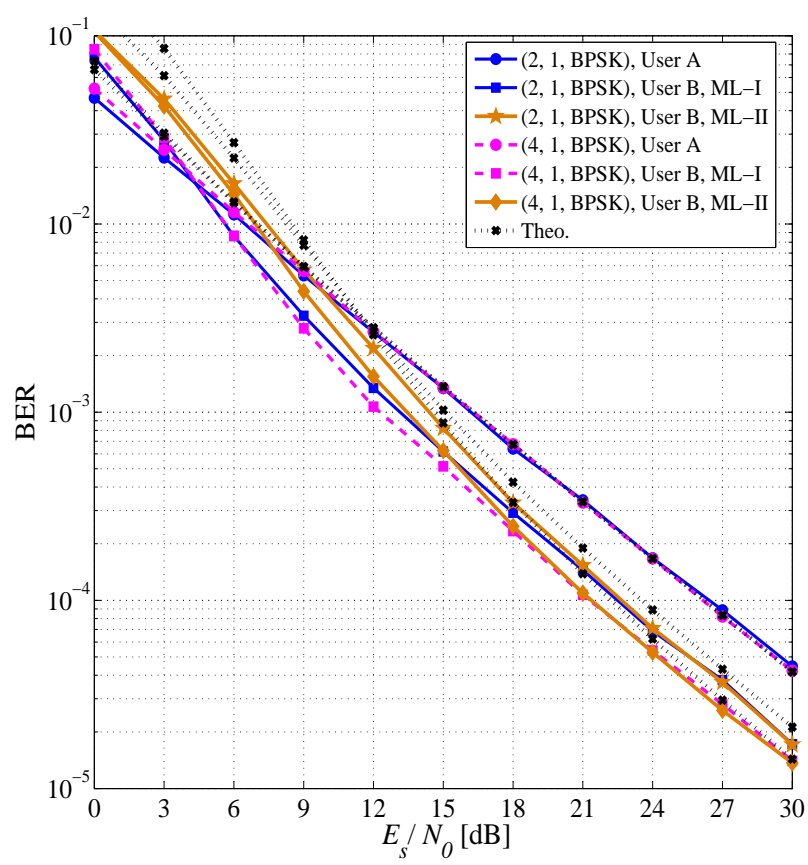

Fig. 3. BER versus $E_{s} / N_{0}$ performance of the CDM-NOMA scheme for numerical verification purposes: different numbers of subcarriers $n$.

and the remaining subcarriers $(n-k)$ are allocated to user A to convey the $M$-PSK signal, and "C-NOMA ( $M_{A}$-PSK, $M_{B}$-PSK)" as the C-NOMA scheme, in which the BS maps the information bits for users A and B into $M_{A}$-PSK and $M_{B^{-}}$ PSK symbols, respectively.

\section{A. Validation of Theoretical Results}

In this subsection, we validate the BER analysis for the proposed schemes with different numbers of subcarriers $n$. The value of power allocation factor $\alpha$ is fixed as 0.22 to study the theoretical BER. Further, for clarity, we refer to the optimal and suboptimal ML detectors as ML-I and ML-II, respectively.

In Fig. 2, we compare the simulative BER results with the theoretical curves obtained from Sections III-A and III-B for GCIM-NOMA, where the ML-I and ML-II detections are employed for user B. First, we can observe from Fig. 2 that the derived BER upper bounds of user A accurately agree with their simulative counterparts in the SNR range of interest with different $n$. On the other hand, for user $\mathrm{B}$, it is clear that the corresponding BER upper bound gradually becomes looser as $n$ goes large, although the theoretical results match well with the simulation counterparts in the high SNR region when $n=4$. This is because that $P_{I B_{2}}$ and $\bar{P}_{I B_{2}}$ from (38) and (39) are excessively enlarged with the increase of $n$. Moreover, we further analyze the impact of different numbers of subcarriers $n$ on the performance of GCIM-NOMA. As anticipated, from Fig. 2, we can observe that the BER performance of the proposed scheme is improved with the increase of $n$ when the other system parameters are fixed. This can be explained by 


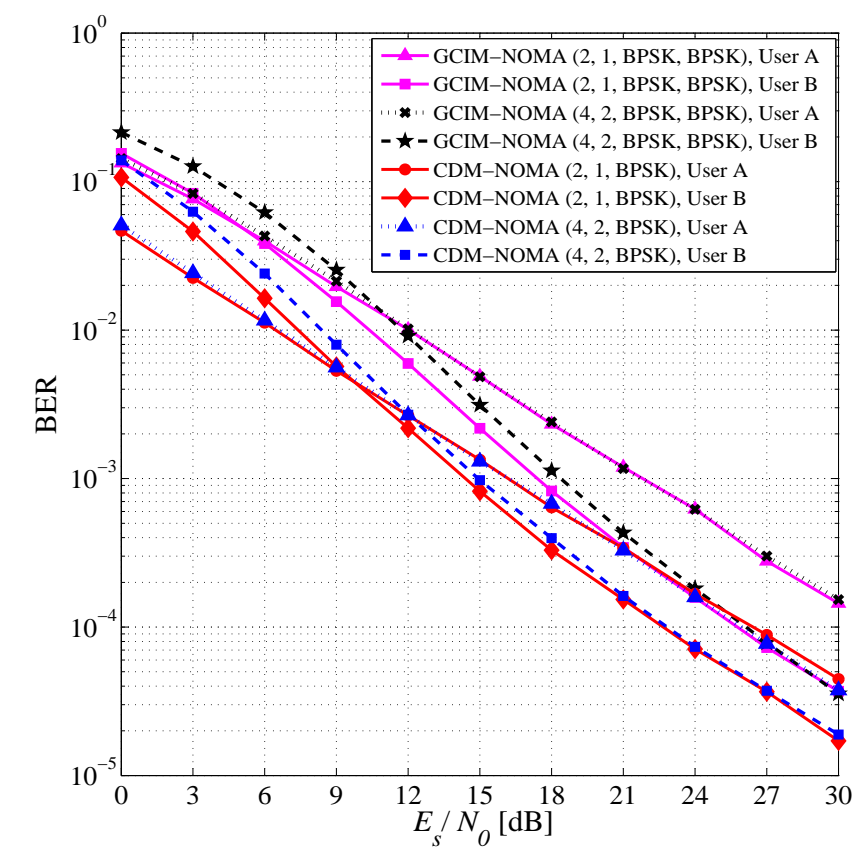

Fig. 4. BER performance comparisons: the SEs of users A and B are 0.5 bpcu and $1 \mathrm{bpcu}$, respectively.

the nature of OFDM-IM that the transmitted power allocated to per activated subcarrier degrades with the decrease of $n$.

In Fig. 3, we investigate the BER performance of the CDM-NOMA scheme, involving the analytical verification and the impact of different $n$ on the system. Obviously, Fig. 3 has shown the validity of the theoretical analysis described perviously in Section III by the asymptotically tight BER upper bounds for users A and B. Additionally, as can be seen from Fig. 3, the performance of user A remains constant, while user B performs better with the increase of $n$. The abovementioned result demonstrates that the spatial informationbearing unit of user B has little interference to user A as only $n$ varies in all system parameters. On the other side, it also implies that the IM bits gradually occupy a larger proportion in the total transmitted bits for user B, which further enhances the performance of user B due to the high reliability of IM bits.

Finally, let us focus on the comparison between the ML-I and ML-II detectors for the proposed schemes. Figs. 2 and 3 clearly illustrate that the ML-II detector has the capability of achieving near-optimal performance for all considered schemes at high SNR, and the performance loss is confined within $1.5 \mathrm{~dB}$ in comparison with the ML-I detector in the low-to-medium SNR regime. Consequently, given the low complexity and reliability of the ML-II detector, it is generally used by default for the proposed schemes unless otherwise specified in the following simulations.

\section{B. GCIM-NOMA v.s. CDM-NOMA}

Now let us use the simulated BER results to demonstrate the achievable BER performance for the proposed schemes. Alternatively, for fairness, in the subsequent simulations, the

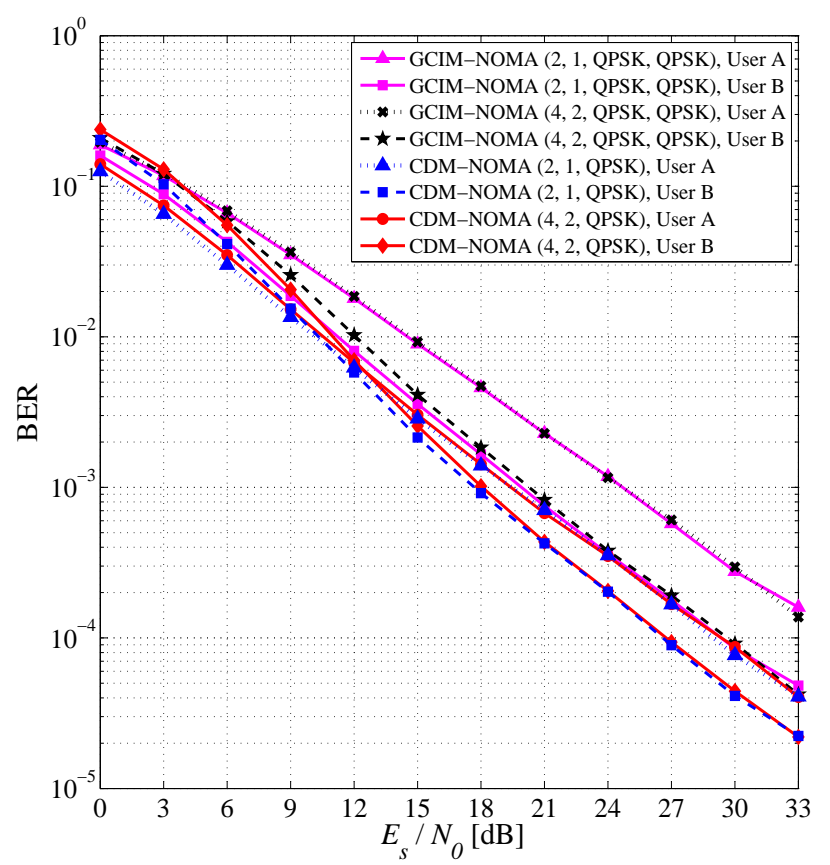

Fig. 5. BER performance comparisons: the SEs of users A and B are $1 \mathrm{bpcu}$ and $1.5 \mathrm{bpcu}$, respectively.

optimal power allocation factor $\alpha$ has been obtained from the exhaustive computer search [13]. In this subsection, we have $\alpha=0.22$.

Figs. 4 and 5 present the BER performance comparison between the proposed GCIM-NOMA and CDM-NOMA when the modulation order $M_{A}\left(M_{B}\right)$ grows from 2 to 4 . In Fig. 4, users $\mathrm{A}$ and $\mathrm{B}$ for all schemes achieve the SEs of 0.5 bits per channel use (bpcu) and $1 \mathrm{bpcu}$, and in Fig. 5, the SEs of 1 bpcu and 1.5 bpcu are obtained for users A and B, respectively. As can be observed from Figs. 4 and 5, both users of CDM-NOMA always outperform those of GCIMNOMA in all simulation scenarios considered. Specifically, in Fig. 4, SNR gains of up to $5 \mathrm{~dB}$ and $3 \mathrm{~dB}$ for users $\mathrm{A}$ and $\mathrm{B}$ are attained at BER $=10^{-4}$. These advantages can be accounted for by three reasons: the first reason is that CDMNOMA conveys the message signal of users A and B with the full transmit power, compared with GCIM-NOMA; the second reason is that the IUI and the imperfect SIC has been greatly alleviated by cancelling power allocation at BS; the last one is that considering all proposed schemes with highly reliable IM bits, GCIM-NOMA has no extra advantage. On the other side, in Fig. 5, it can be found that significant SNR gains are also achieved by CDM-NOMA over GCIM-NOMA, i.e., about $5 \mathrm{~dB}$ and $3 \mathrm{~dB}$ for users $\mathrm{A}$ and $\mathrm{B}$ at $\mathrm{BER}=10^{-4}$. This means that with the increase of $M$, the superiority of CDMNOMA still holds. Alternatively, it can be figured out from Figs. 4 and 5 that the performance gap among users of CDMNOMA is always smaller than that of GCIM-NOMA when different system configurations are considered, which shows that CDM-NOMA is capable of attaining better user fairness than GCIM-NOMA. 


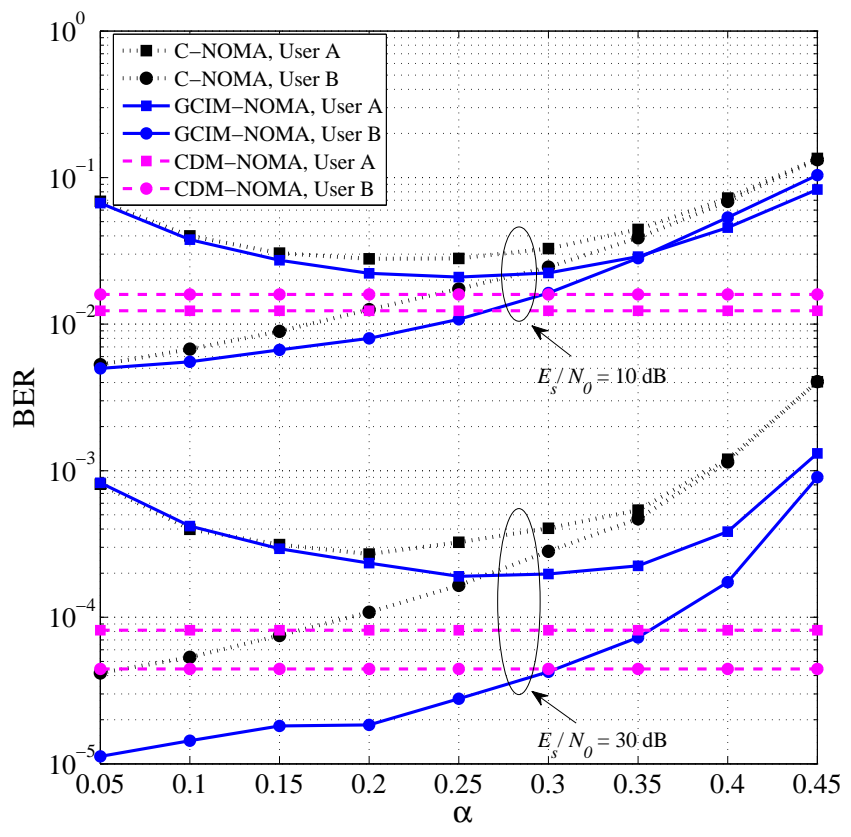

Fig. 6. BER performance comparisons among "GCIM-NOMA (4, 2, QPSK, BPSK)", "CDM-NOMA (4, 2, QPSK)", and "C-NOMA (BPSK, BPSK)" with different power allocation coefficient.

\section{Proposed Schemes v.s. C-NOMA}

Considering the existence of power allocation mechanism in GCIM-NOMA and C-NOMA, we perform the corresponding simulation to uncover the influence of power allocation coefficient $\alpha$ on the BER performances of users for all considered schemes, as shown in Fig. 6. Meanwhile, to ensure the comparison fairness, we set the $\mathrm{SE}$ of users $\mathrm{A}$ and $\mathrm{B}$ as 1 bpcu for all schemes. In order to study the impact of SNR on these schemes, we give different values of SNR, i.e., $E_{s} / N_{0}$ $=10 \mathrm{~dB}, 30 \mathrm{~dB}$. First, in Fig. 6, it can be observed that the BER performance of CDM-NOMA remains unchanged with the increase of $\alpha$, due to the lack of power allocation. As for user A, the BER performance of CDM-NOMA is always superior to that of C-NOMA; while for user B, C-NOMA with an extremely smaller value of $\alpha$ has the capability to outperform CDM-NOMA, as SNR goes large. However, this approach is unreasonable and impractical, because it causes the fairness loss of the holistic system. Furthermore, let us focus on the comparison between GCIM-NOMA and C-NOMA. From Fig. 6, we can know that GCIM-NOMA always performs better, and the performance gain becomes significant with the increase of SNR. This can be interpreted as that with the introduction of IM, a high-reliability information-bearing unit is produced, and it is employed to carry the message of the cell-edge user, thus effectively mitigating the impact of IUI and improving the system performance. Finally, it is interesting to investigate the comparison between the two proposed schemes. In contrast with GCIM-NOMA, CDM-NOMA can further guarantee the fairness among users and attain acceptable performance, but it also exhibits less flexibility than GCIM-NOMA to in coordinating the performance of users.

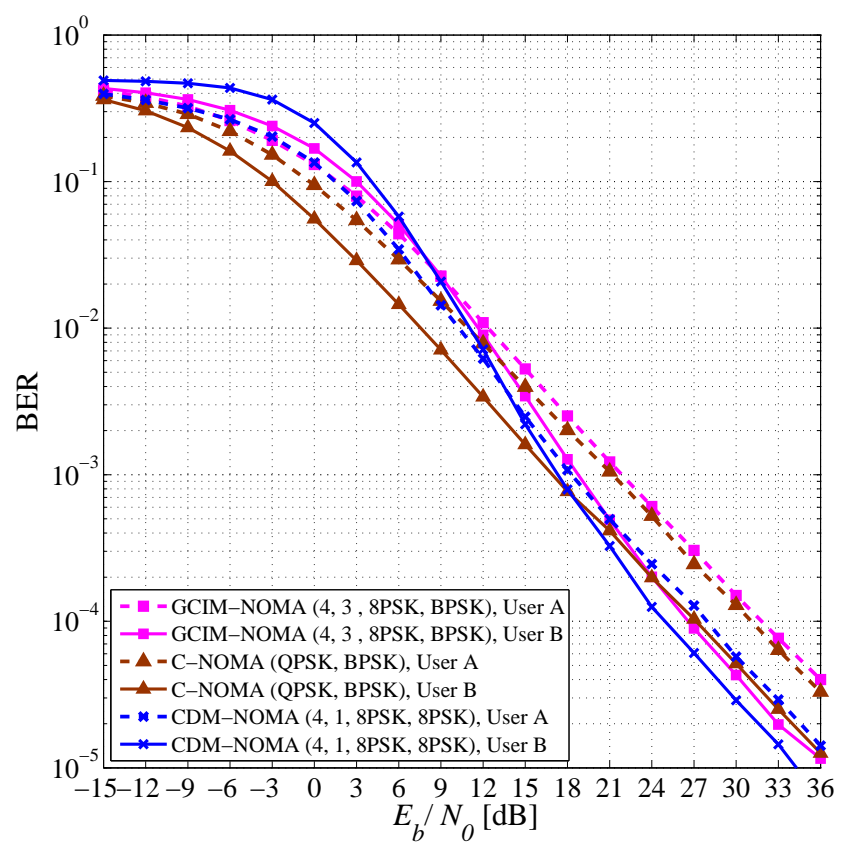

Fig. 7. BER performance comparisons: "GCIM-NOMA (4, 3, 8PSK, BPSK)", "CDM-NOMA (4, 1, 8PSK)", and "C-NOMA (QPSK, BPSK)".

In Fig 7, in order to comprehensively analyze the difference among the two proposed schemes and C-NOMA, we further compare them according to the following simulation configurations, i.e.,

1) "GCIM-NOMA (4, 3, 8PSK, BPSK)" with the SE of users $\mathrm{A}$ and $\mathrm{B}$ equal to $2.25 \mathrm{bpcu}$ and $1.25 \mathrm{bpcu}$, respectively;

2) "CDM-NOMA (4, 1, 8PSK)" with the SE of users A and B equal to $2.25 \mathrm{bpcu}$ and $1.25 \mathrm{bpcu}$, respectively,

where "C-NOMA (QPSK, BPSK)" is used as the benchmark. Moreover, note that the optimal values of $\alpha$ are 0.29 and 0.38 for C-NOMA and GCIM-NOMA, respectively. As shown in Fig. 7, on one hand, we can find that CDM-NOMA exhibits the best performance among all considered schemes. Specifically, compared with C-NOMA, SNR gains of about $3 \mathrm{~dB}$ and $2 \mathrm{~dB}$ are attained for users $\mathrm{A}$ and $\mathrm{B}$, respectively, which is mainly due to the utilization of two independent information-bearing units and the elimination of error propagation of SIC. On the other hand, it is clear that there is no significant difference in the performance of GCIM-NOMA and C-NOMA at the high SNR region, which suggests that the limited transmission rate is the bottleneck of GCIM-NOMA.

\section{CONClusion}

In this paper, we have proposed the GCIM-NOMA and CDM-NOMA schemes, in which the techniques of C-NOMA and IM are integrated based on the OFDM framework to further improve the system performance in terms of BER and SE. The basic principle of GCIM-NOMA is to allocate IM bits to serve the cell-edge user, and save the transmit power to further assist the transmission of the superposed 
signal to users. Meanwhile, during the cooperative phase, the cell-edge user receives the OFDM-IM signal with its information regenerated at the cell-center user. Furthermore, CDM-NOMA, as an extension of GCIM-NOMA, has been proposed for eliminating the user interference and ensuring a high transmission rate. In this scheme, different modulation modes (Mode I and Mode II) are employed to distinguish whether the OFDM subcarriers carry the information for the cell-center user or the cell-edge one, so as to avoid the overlap of multi-user information in the power domain. Alternatively, this method can generate IM bits with high reliability, which provides better services to the cell-edge user. To effectively reduce the higher computational complexity of the optimal ML detector, we have also designed a lowcomplexity sub-optimal ML detector for the cell-edge user in the proposed GCIM-NOMA and CDM-NOMA systems. Both analytical derivations and numerical simulations have proven that the proposed GCIM-NOMA and CDM-NOMA schemes considerably outperform the classical C-NOMA system.

\section{APPENDIX A}

\section{DERIVATION OF (28)}

According to the well-known union bound technique, $P_{A_{1}}$ can be upper bounded by

$$
\begin{aligned}
P_{A_{1}} \leq & \frac{1}{p_{2}^{A} 2^{p}} \sum_{I} \sum_{\hat{I}, I \neq \hat{I}} \operatorname{Pr}(I \rightarrow \hat{I}) \\
& \sum_{\mathbf{s}_{B}, \hat{\mathbf{s}}_{B}} \sum_{\mathbf{s}_{A}, \hat{\mathbf{s}}_{A}} \operatorname{Pr}(\mathbf{x} \rightarrow \hat{\mathbf{x}} \mid I \rightarrow \hat{I}) N\left(\mathbf{s}_{A}, \hat{\mathbf{s}}_{A}\right),
\end{aligned}
$$

where $N\left(\mathbf{s}_{A}, \hat{\mathbf{s}}_{A}\right)$ represents the number of erroneous bits when $\mathbf{s}_{A}$ is detected as $\hat{\mathbf{s}}_{A}$, and $\operatorname{Pr}(\mathbf{x} \rightarrow \hat{\mathbf{x}} \mid I \rightarrow \hat{I})$ means the error probability of detecting the transmitted $\mathbf{x}$ as $\hat{\mathbf{x}}$ in case of the detection error for the index of active subcarrier $I \rightarrow \hat{I}$. Given that $\mathbf{x}$ is created through combining $\mathbf{s}_{A}, \mathbf{s}_{B}$ and $I$, (44) can be rewritten as

$$
\begin{aligned}
P_{A_{1}} \leq & \frac{1}{p_{2}^{A} 2^{p}} \sum_{I} \sum_{\hat{I}, I \neq \hat{I}} \operatorname{Pr}(I \rightarrow \hat{I}) \\
& \sum_{\mathbf{s}_{B}, \hat{\mathbf{s}}_{B}} \sum_{\mathbf{s}_{A}, \hat{\mathbf{s}}_{A}} \operatorname{Pr}\left(\mathbf{s}_{B} \rightarrow \hat{\mathbf{s}}_{B}, \mathbf{s}_{A} \rightarrow \hat{\mathbf{s}}_{A} \mid I \rightarrow \hat{I}\right) N\left(\mathbf{s}_{A}, \hat{\mathbf{s}}_{A}\right) .
\end{aligned}
$$

Based on the Bayesian rule, $\operatorname{Pr}\left(\mathbf{s}_{B} \rightarrow \hat{\mathbf{s}}_{B}, \mathbf{s}_{A} \rightarrow \hat{\mathbf{s}}_{A} \mid I \rightarrow \hat{I}\right)$ can be further decomposed as

$$
\begin{aligned}
& \operatorname{Pr}\left(\mathbf{s}_{B} \rightarrow \hat{\mathbf{s}}_{B}, \mathbf{s}_{A} \rightarrow \hat{\mathbf{s}}_{A} \mid I \rightarrow \hat{I}\right) \\
& =\operatorname{Pr}\left(\mathbf{s}_{B} \rightarrow \hat{\mathbf{s}}_{B} \mid I \rightarrow \hat{I}\right) \operatorname{Pr}\left(\mathbf{s}_{A} \rightarrow \hat{\mathbf{s}}_{A} \mid I \rightarrow \hat{I}, \mathbf{s}_{B} \rightarrow \hat{\mathbf{s}}_{B}\right) .
\end{aligned}
$$

Substituting (46) into (45), we can rewrite (45) as (47), shown on the top of the next page, where

$$
\begin{aligned}
T(\cdot)= & N\left(s_{A, \cdot}, \hat{s}_{A, \cdot}\right) \operatorname{Pr}\left(s_{B, \cdot} \rightarrow \hat{s}_{B, \cdot} \mid I \rightarrow \hat{I}\right) \\
& \times \operatorname{Pr}\left(s_{A, \cdot} \rightarrow \hat{s}_{A, \cdot} \mid I \rightarrow \hat{I}, s_{B, \cdot} \rightarrow \hat{s}_{B, \cdot}\right) .
\end{aligned}
$$

Relying on the $k$ activated subcarriers out of $n$ available ones, we also have $|\tau \notin I \cap \hat{I}, \tau \in I \cup \hat{I}|=\bar{D}(I, \hat{I})$ and $|m \in I \cap \hat{I}|=D(I, \hat{I})$. For brevity, we define

$$
\mathcal{J}_{1}=\sum_{m \in I \cap \hat{I}} \frac{1}{p_{2}^{A} 2^{p_{2}}} \sum_{\mathbf{s}_{B}, \hat{\mathbf{s}}_{B}} \sum_{\mathbf{s}_{A}, \mathbf{s}_{A}} T(m),
$$

and

$$
\mathcal{J}_{2}=\sum_{\tau \notin I \cap \hat{I}, \tau \in I \cup \hat{I}} \frac{1}{p_{2}^{A} 2^{p_{2}}} \sum_{\mathbf{s}_{B}, \hat{\mathbf{s}}_{B}} \sum_{\mathbf{s}_{A}, \hat{\mathbf{s}}_{A}} T(\tau) .
$$

According to the fact described previously that the bit errors of user A also depend on whether the SIC process is detected correctly, we can further decompose $\mathcal{J}_{1}$ as

$$
\begin{aligned}
\mathcal{J}_{1}=\frac{1}{p_{2}^{A} 2^{p_{2}}} \sum_{\mathbf{s}_{B}, \hat{\mathbf{s}}_{B}} \sum_{\mathbf{s}_{A}, \hat{\mathbf{s}}_{A}} \sum_{m \in I \cap \hat{I}}\left\{\sum_{s_{B, m}=\hat{s}_{B, m}} T(m)\right. & \left.+\sum_{s_{B, m} \neq \hat{s}_{B, m}} T(m)\right\} \\
=\sum_{m \in I \cap \hat{I}}\{\zeta(m)+\bar{\zeta}(m)\}, &
\end{aligned}
$$

where

$$
\zeta(m)=\frac{1}{2^{p_{2}^{B}}} \sum_{\mathbf{s}_{B}, \hat{\mathbf{s}}_{B}} \sum_{s_{B, m}=\hat{s}_{B, m}} \frac{1}{p_{2}^{A} 2^{p_{2}^{B}}} \sum_{\mathbf{s}_{A}, \hat{\mathbf{s}}_{A}} T(m)
$$

and

$$
\bar{\zeta}(m)=\frac{1}{2^{p_{2}^{B}}} \sum_{\mathbf{s}_{B}, \hat{\mathbf{s}}_{B}} \sum_{s_{B, m} \neq \hat{s}_{B, m}} \frac{1}{p_{2}^{A} 2^{p_{2}^{B}}} \sum_{\mathbf{s}_{A}, \hat{\mathbf{s}}_{A}} T(m)
$$

are associated with the subcarrier whose SIC processes are detected correctly or incorrectly conditioned on $m \in\{I \cap \hat{I}\}$, respectively. It can be figured out that $\zeta(m)$ is the error probability of $M_{A}$-PSK when the SIC procedure has been performed correctly. By substituting (48) into (52), $\zeta(m)$ can be expressed as

$$
\zeta(m)=\frac{1}{2^{p_{2}^{B}}} \sum_{\mathbf{s}_{B}, \hat{\mathbf{s}}_{B}} \sum_{s_{B}, m=\hat{s}_{B, m}} P_{1} \operatorname{Pr}\left(s_{B, m} \rightarrow \hat{s}_{B, m} \mid I \rightarrow \hat{I}\right),
$$

where

$$
\begin{aligned}
P_{1}= & \frac{1}{p_{2}^{A} 2^{p_{2}^{A}}} \sum_{\mathbf{s}_{A}, \hat{\mathbf{s}}_{A}} N\left(s_{A, m}, \hat{s}_{A, m}\right) \\
& \operatorname{Pr}\left(s_{A, m} \rightarrow \hat{s}_{A, m} \mid I \rightarrow \hat{I}, s_{B, m} \rightarrow \hat{s}_{B, m}\right)
\end{aligned}
$$

is the BER upper bound of $M_{A}$-PSK obtained from the union bound technique. Therefore, we have $P_{1} \approx P_{A b}\left(M_{A}\right)$ in the high SNR region. Meanwhile, it is easily proven that

$$
1-P_{S I C} \approx \frac{1}{2^{p_{2}^{B}}} \sum_{\mathbf{s}_{B}, \hat{\mathbf{s}}_{B}} \sum_{s_{B, m}=\hat{s}_{B, m}} \operatorname{Pr}\left(s_{B, m} \rightarrow \hat{s}_{B, m} \mid I \rightarrow \hat{I}\right) .
$$

Based on the above-mentioned derivations, we can rewrite (54) as

$$
\zeta(m) \approx\left(1-P_{S I C}\right) P_{A b}\left(M_{A}\right) .
$$

On the other hand, it can be found that $\bar{\zeta}(m)$ means the error event with the incorrect SIC process. Consequently, following the derivation of (52), $\bar{\zeta}(m)$ can be calculated as

$$
\bar{\zeta}(m) \approx \frac{1}{2} P_{S I C}
$$

Substituting (57) and (58) into (51), $\mathcal{J}_{1}$ can be rewritten as

$$
\mathcal{J}_{1}=D(I, \hat{I})\left\{\left(1-P_{S I C}\right) P_{A b}+\frac{1}{2} P_{S I C}\right\} .
$$




$$
\begin{aligned}
P_{A_{1}} & \leq \frac{1}{p_{2}^{(A)} 2^{p}} \sum_{I} \sum_{\hat{I}, I \neq \hat{I}} \operatorname{Pr}(I \rightarrow \hat{I}) \sum_{\mathbf{s}_{B}, \hat{\mathbf{s}}_{B}} \sum_{\mathbf{s}_{A}, \hat{\mathbf{s}}_{A}} \sum_{\alpha \in I \cup \hat{I}} T(\alpha) \\
& =\frac{1}{2^{p_{1}}} \sum_{I} \sum_{\hat{I}, I \neq \hat{I}} \operatorname{Pr}(I \rightarrow \hat{I}) \frac{1}{p_{2}^{A} 2^{p_{2}}} \sum_{\mathbf{s}_{B}, \hat{\mathbf{s}}_{B}} \sum_{\mathbf{s}_{A}, \hat{\mathbf{s}}_{A}}\left[\sum_{m \in I \cap \hat{I}} T(m)+\sum_{\tau \notin I \cap \hat{I}, \tau \in I \cup \hat{I}} T(\tau)\right] \\
& =\frac{1}{2^{p_{1}}} \sum_{I} \sum_{\hat{I}, I \neq \hat{I}} \operatorname{Pr}(I \rightarrow \hat{I})\left[\sum_{m \in I \cap \hat{I}} \frac{1}{p_{2}^{A} 2^{p_{2}}} \sum_{\mathbf{s}_{B}, \hat{\mathbf{s}}_{B}} \sum_{\mathbf{s}_{A}, \hat{\mathbf{s}}_{A}} T(m)+\sum_{\tau \notin I \cap \hat{I}, \tau \in I \cup \hat{I}} \frac{1}{p_{2}^{A} 2^{p_{2}}} \sum_{\mathbf{s}_{B}, \hat{\mathbf{s}}_{B}} \sum_{\mathbf{s}_{A}, \hat{\mathbf{s}}_{A}} T(\tau)\right] .
\end{aligned}
$$

Furthermore, as for $\mathcal{J}_{2}$, considering that the activated subcarrier is detected erroneously in this case, it is reasonable to let

$$
\mathcal{J}_{2}=\frac{1}{2} \bar{D}(I, \hat{I})
$$

Finally, (28) can be obtained from (47), (59) and (60).

\section{REFERENCES}

[1] S. K. Haider, A. Jiang, M. A. Jamshed, H. Pervaiz, and S. Mumtaz, "Performance enhancement in P300 ERP single trial by machine learning adaptive denoising mechanism," IEEE Netw. Lett., vol. 1, no. 1, pp. 26-29, Mar. 2019.

[2] Z. Zheng, T. Wang, J. Wen, S. Mumtaz, A. K. Bashir, and S. H. Chauhdary, "Differentially private high-dimensional data publication in internet of things," IEEE Internet Things J., Nov. 2019.

[3] J. G. Andrews et al., "What will 5G be?" IEEE J. Sel. Areas Commun., vol. 32, no. 6, pp. 1065-1082, Jun. 2014.

[4] S. Timotheou and I. Krikidis, "Fairness for non-orthogonal multiple access in 5G systems," IEEE Signal Process. Lett., vol. 22, no. 10, pp. 1647-1651, Oct. 2015.

[5] Z. Ding, Z. Yang, P. Fan, and H. V. Poor, "On the performance of non-orthogonal multiple access in $5 \mathrm{G}$ systems with randomly deployed users," IEEE Signal Process. Lett., vol. 21, no. 12, pp. 1501-1505, Dec. 2014.

[6] Z. Ding, M. Peng, and H. V. Poor, "Cooperative non-orthogonal multiple access in 5G systems," IEEE Commun. Lett., vol. 19, no. 8, pp. 14621465, Aug. 2015.

[7] Y. Liu, Z. Ding, M. Elkashlan, and H. V. Poor, "Cooperative nonorthogonal multiple access with simultaneous wireless information and power transfer," IEEE J. Sel. Areas Commun., vol. 34, no. 4, pp. 938953, Apr. 2016.

[8] J. Kim and I. Lee, "Capacity analysis of cooperative relaying systems using non-orthogonal multiple access," IEEE Commun. Lett., vol. 19, no. 11, pp. 1949-1952, Nov. 2015.

[9] M. Xu, F. Ji, M. Wen, and W. Duan, "Novel receiver design for the cooperative relaying system with non-orthogonal multiple access," IEEE Commun. Lett., vol. 20, no. 8, pp. 1679-1682, Aug. 2016.

[10] F. Kara and H. Kaya, "On the error performance of cooperative-NOMA with statistical CSIT," IEEE Commun. Lett., vol. 23, no. 1, pp. 128-131, Jan. 2019.

[11] R. Jiao, L. Dai, J. Zhang, R. MacKenzie, and M. Hao, "On the performance of NOMA-based cooperative relaying systems over Rician fading channels," IEEE Trans. Veh. Technol., vol. 66, no. 12, pp. 1140911413, Dec. 2017

[12] T. Nguyen and X. Tran, "Performance of cooperative NOMA system with a full-duplex relay over Nakagami-m fading channels," in Proc IEEE Intern. Conf. Recent Advances Signal Process., Telecommun. and Computing (SigTelCom), Hanoi, Vietnam, Mar. 2019, pp. 130-134.

[13] Q. Li, M. Wen, E. Basar, H. V. Poor, and F. Chen, "Spatial modulationaided cooperative NOMA: Performance analysis and comparative study," IEEE J. Sel. Topics Signal Process., vol. 13, no. 3, pp. 715-728, June 2019.
[14] L. Pan and J. Zheng, "Spatial modulation aided cooperative NOMA: Implementation and achievable rate analysis," IEEE Intern. Conf. Commun. (ICC), Shanghai, China, May 2019, pp. 1-6.

[15] E. Basar, M. Wen, R. Mesleh, M. Di Renzo, Y. Xiao, and H. Haas, "Index modulation techniques for next-generation wireless networks," IEEE Access, vol. 5, pp. 16693-16746, Aug. 2017.

[16] M. Wen, X. Cheng, and L. Yang, Index Modulation for $5 G$ Wireless Communications. Berlin, Germany: Springer, 2017.

[17] T. Mao, Q. Wang, Z. Wang, and S. Chen, "Novel index modulation techniques: A survey," IEEE Commun. \& Surveys Tuts., vol. 21, no. 1, pp. 315-348, Firstquarter 2019.

[18] E. Basar, U. Ayglu, E. Panayirci, and H. V. Poor, "Orthogonal frequency division multiplexing with index modulation," IEEE Trans. Signal Process., vol. 61, no. 22, pp. 5536-5549, Nov. 2013.

[19] J. Li, Y. Peng, Y. Yan, X. Jiang, H. Hai, and M. Zukerman, "Cognitive radio network assisted by OFDM with index modulation," IEEE Trans. Veh. Technol., vol. 69, no. 1, pp. 1106-1110, Jan. 2020.

[20] M. Wen, B. Ye, E. Basar, Q. Li, and F. Ji, "Enhanced orthogonal frequency division multiplexing with index modulation," IEEE Trans. Wireless Commun., vol. 16, no. 7, pp. 4786-4801, July 2017.

[21] T. Mao, Z. Wang, Q. Wang, S. Chen, and L. Hanzo, "Dual-mode index modulation aided OFDM," IEEE Access, vol. 5, pp. 50-60, Feb. 2017.

[22] T. Mao, Q. Wang, and Z. Wang, "Generalized dual-mode index modulation aided OFDM," IEEE Commun. Lett., vol. 21, no. 4, pp. 761-764, Apr. 2017.

[23] S. Dogan, A. Tusha, and H. Arslan, "NOMA with index modulation for uplink URLLC through grant-free access," IEEE J. Sel. Topic. Signal Process., vol. 13, no. 6, pp. 1249-1257, Oct. 2019.

[24] E. Chatziantoniou, Y. Ko, and J. Choi, "Non-orthogonal multiple access with multi-carrier index keying," in Proc. European Wireless Conf., Dresden, Germany, May 2017, pp. 1-5.

[25] Z. Ding, Z. Yang, P. Fan, and H. V. Poor, "On the performance of non-orthogonal multiple access in 5G systems with randomly deployed users," IEEE Signal Process. Lett., vol. 21, no. 12, pp. 1501-1505, Dec. 2014.

[26] M. Wen, Q. Li, E. Basar, and W. Zhang, "Generalized multiple-mode OFDM with index modulation," IEEE Trans. Wireless Commun., vol. 17, no. 10, pp. 6531-6543, Oct. 2018.

[27] Q. Li, M. Wen, H. V. Poor, and F. Chen, "Information guided precoding for OFDM," IEEE Access, vol. 5, pp. 19644-19656, Oct. 2017.

[28] M. Chiani and D. Dardari, "Improved exponential bounds and approximation for the Q-function with application to average error probability computation," IEEE Global Telecommun. Conf., Taiwan, Nov. 2002, pp. 1399-1402

[29] F. Kara and H. Kaya, "BER performances of downlink and uplink NOMA in the presence of SIC errors over fading channels," IET Commun., vol. 12, no. 15, pp. 1834-1844, Sept. 2018.

[30] M. K. Simon and M.-S. Alouini, Digital Communication Over Fading Channels, 2nd ed. New York, NY, USA: Wiley, 2005.

[31] John Proakis, Digital Communications, 4th Ed. New York, NY, USA:Mc-Graw-Hill, 2000

[32] J. Crawford, E. Chatziantoniou, and Y. Ko, "On the SEP analysis of OFDM index modulation with hybrid low complexity greedy detection and diversity reception," IEEE Trans. Veh. Technol., vol. 66, no. 9, pp. 8103-8118, Sept. 2017. 


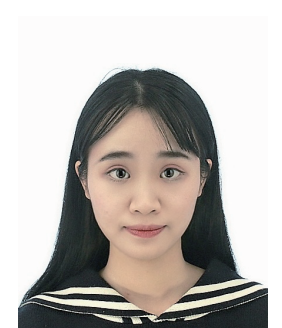

Xuan Chen received the B.S. degree from Wuhan University of Technology, Wuhan, China, in 2017. She is currently pursuing the Ph.D. degree with the South China University of Technology, Guangzhou, China. In August 2019, she was a visiting student for molecular communication in Yonsei University, Seoul, South Korea.Her main research interests include index modulation and molecular communications.

She was the winner in data bakeoff competition (Molecular MIMO) at IEEE Communication Theory Workshop (CTW) 2019, Selfoss, Iceland.

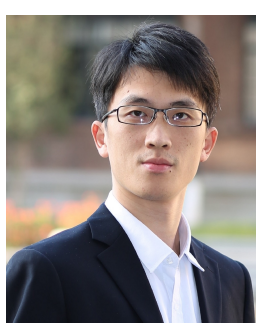

Miaowen Wen (SM'18) received the Ph.D. degree from Peking University, Beijing, China, in 2014. From 2012 to 2013, he was a Visiting Student Research Collaborator with Princeton University, Princeton, NJ, USA. He is currently an Associate Professor with South China University of Technology, Guangzhou, China, and a Hong Kong Scholar with The University of Hong Kong, Hong Kong. He has published a Springer book entitled Index Modulation for $5 G$ Wireless Communications and more than 80 journal papers. His research interests include a variety of topics in the areas of wireless and molecular communications.

Dr. Wen was the recipient of four Best Paper Awards from the IEEE ITST'12, the IEEE ITSC'14, the IEEE ICNC'16, and the IEEE ICCT'19. He was recognized as an Exemplary Reviewer for the IEEE COMMUNICATIONS LETTERS in 2017 and the IEEE TRANSACTIONS ON COMMUNICATIONS in 2019. He has served on the Editorial Boards of the IEEE ACCESS, and the EURASIP Journal on Wireless Communications and Networking, and a Guest Editor for IEEE JOURNAL ON SELECTED AREAS IN COMMUNICATIONS (Special Issue on Spatial Modulation for Emerging Wireless Systems) and for IEEE Journal of SElEcted Topics In SIgnal Processing (Special Issue on Index Modulation for Future Wireless Networks: A Signal Processing Perspective). Currently, he is serving as an Editor for the IEEE TRANSACTIONS ON COMMUNICATIONS, the IEEE COMMUNICATIONS LETTERS, and the Physical Communication (Elsevier).

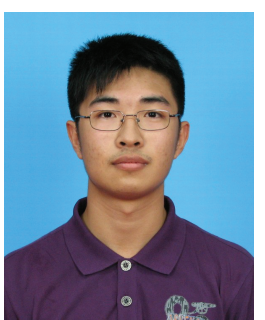

Tianqi Mao received the B.S. and M.S. degrees (with Highest Hons.) in electronic engineering from Tsinghua University, Beijing, China, in 2015 and 2018, respectively, where he is currently pursuing the Ph.D. degree with the Department of Electronic Engineering at Tsinghua University.

His current research interests include modulation and signal processing for wireless communication, terahertz $(\mathrm{THz})$ wireless communication and visible light communication. He was a recipient of the Science and Technology Award (Second Prize) of China Institute of Communications (CIC), the Excellent Master Dissertation of Chinese Institute of Electronics (CIE), the Special Scholarship of Tsinghua University, the Outstanding Master Graduate of Tsinghua University, the Outstanding Master Graduate of Beijing City, the Excellent Master Dissertation of Tsinghua University, and National Scholarship.

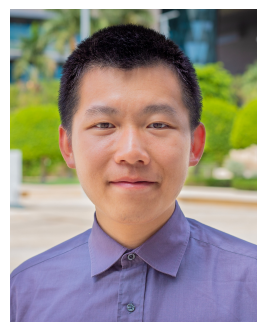

Shuping Dang (S'13-M'18) received B.Eng (Hons) in Electrical and Electronic Engineering from the University of Manchester (with first class honors) and B.Eng in Electrical Engineering and Automation from Beijing Jiaotong University in 2014 via a joint ' $2+2$ ' dual-degree program. He also received D.Phil in Engineering Science from University of Oxford in 2018. Dr. Dang joined in the R\&D Center, Huanan Communication Co., Ltd. after graduating from University of Oxford and is currently working as a Postdoctoral Fellow with the Computer, Electrical and Mathematical Science and Engineering Division, King Abdullah University of Science and Technology (KAUST). He is a co-recipient of the "best paper' award for work presented at 2019 19th IEEE International Conference on Communication Technology. He serves as a reviewer for a number of key journals in communications and information science, including IEEE TRANSACTIONS ON WIRELESS COMMUNICATIONS, IEEE TRANSACTIONS ON COMMUNICATIONS, IEEE WiRELESS COMMUNICATIONS LETTERS, IEEE COMMUNICATIONS LETTERS, and IEEE TRANSACTIONS ON VEHICULAR TeChNology. Dr. Dang is recognized as the Exemplary Reviewer of IEEE Communications Letters in 2019. His current research interests include novel modulation schemes, cooperative communications, terahertz communications, and $6 \mathrm{G}$ wireless network design. 